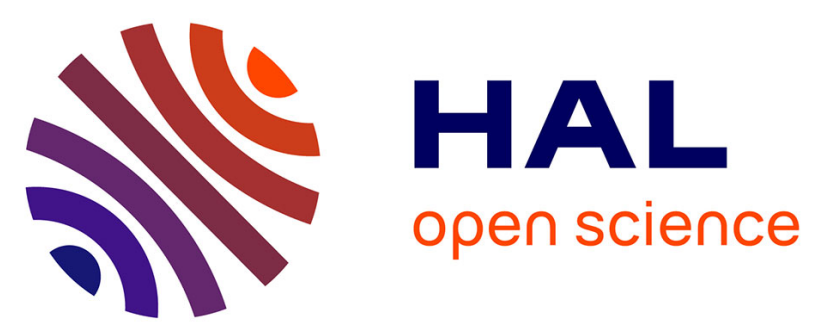

\title{
Preparation of Chiral Photosensitive Organocatalysts and Their Application for the Enantioselective Synthesis of 1,2-Diamines
}

Jiyuan Lyu, Aurélie Claraz, Maxime Vitale, Clémence Allain, Geraldine Masson

\section{To cite this version:}

Jiyuan Lyu, Aurélie Claraz, Maxime Vitale, Clémence Allain, Geraldine Masson. Preparation of Chiral Photosensitive Organocatalysts and Their Application for the Enantioselective Synthesis of 1,2Diamines. Journal of Organic Chemistry, 2020, 85 (20), pp.12843-12855. 10.1021/acs.joc.0c01931 . hal-03009598

\section{HAL Id: hal-03009598 https://hal.science/hal-03009598}

Submitted on 20 Nov 2020

HAL is a multi-disciplinary open access archive for the deposit and dissemination of scientific research documents, whether they are published or not. The documents may come from teaching and research institutions in France or abroad, or from public or private research centers.
L'archive ouverte pluridisciplinaire HAL, est destinée au dépôt et à la diffusion de documents scientifiques de niveau recherche, publiés ou non, émanant des établissements d'enseignement et de recherche français ou étrangers, des laboratoires publics ou privés. 


\section{Preparation of Chiral Photosensitive Organocatalysts and their}

\section{Application for the Enantioselective Synthesis of 1,2-diamines}

Jiyuan Lyu, ${ }^{*}$ Aurélie Claraz, ${ }^{\dagger}$ Maxime R. Vitale, Clémence Allain,,${ }^{+}$and Géraldine Masson $*{ }^{*}$

${ }^{\dagger}$ Institut de Chimie des Substances Naturelles, Université Paris Saclay, CNRS, UPR2301, 1 Avenue de la Terrasse, 91198 Gif-sur-Yvette cedex, France.

† Laboratoire des biomolécules, LBM, Département de chimie, École normale supérieure, PSL University, Sorbonne Université, CNRS, 75005 Paris, France.

*PSM, ENS Paris-Saclay, CNRS UMR 8531, 61, Avenue du Président Wilson, 94235 Cachan cedex, France.

\section{ABSTRACT}

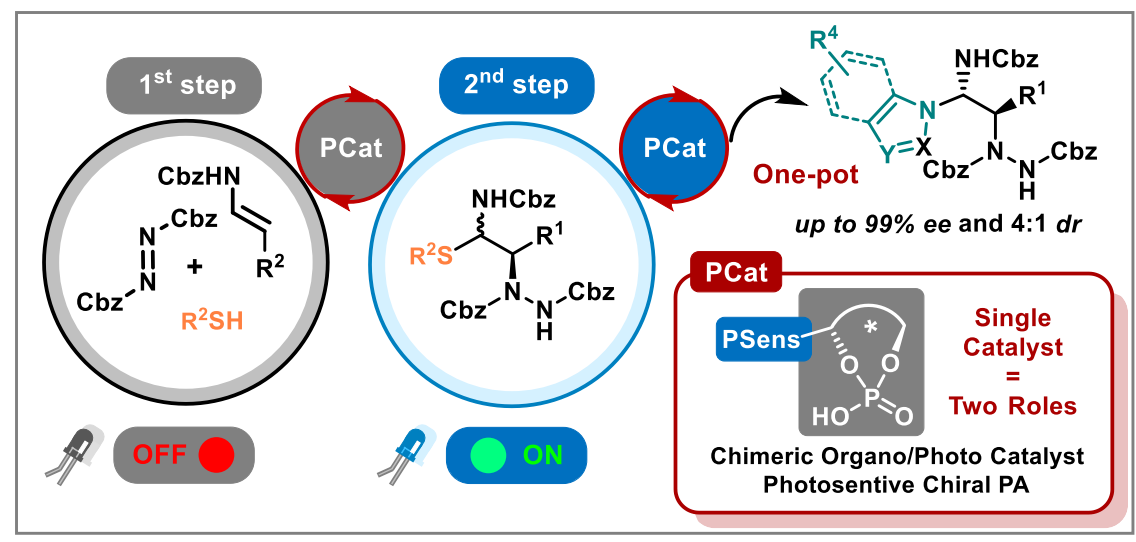

Chiral phosphoric acid based organocatalysis and visible-light photocatalysis have both emerged as promising technologies for the sustainable production of fine chemicals. In this context, we have envisioned the design and the synthesis of a new class of chimeric catalytic entities that would feature both catalytic capabilities. Given their multitask nature, such catalysts would reveal 
particularly attractive for the development of new catalytic transformations, tandem processes in particular. To this aim, several BINOL-based chiral phosphoric acids backbone presenting one or two visible-light sensitive thioxanthone moieties have been prepared and studied. The utility of these new photoactive chiral organocatalysts is then demonstrated in the enantioselective tandem three-component electrophilic amination of enecarbamates. Of note, the $C_{1}$-symmetric organo/photo catalyst has given a better catalytic activity than those presenting a $C_{2}$ symmetry.

\section{INTRODUCTION}

Over the past 50 years, catalysis has become one of the best answers to the sustainability challenge raised by the ever-increasing need for raw and fine chemicals. In this context, different catalytic strategies have been explored, among which organocatalysis has emerged as an especially viable option for the asymmetric production of enantiopure compounds. For instance, thanks to the seminal work of Akiyama and Terada, chiral BINOL-derived phosphoric have been instrumental in the development of highly efficient bifunctional $\mathrm{H}$-bonding catalysis. ${ }^{1}$ Meanwhile, visible-light photoredox catalysis ${ }^{2}$ has arose as an appealing "green" strategy, inasmuch as it allows to harness what certainly is the most inexpensive, abundant, and environmentally benign source of energy: solar energy. By relying on metal-based ${ }^{2 a}$ or purely organic photosensitive entities, ${ }^{2 b, d, e, h}$ a wide array of chemical transformations has been achieved under mild reaction conditions, either exploiting the capacity of such catalysts to induce redox processes or to promote energy transfers. Consequently, intertwining visible light-activated chemistry with asymmetric organocatalysis has been the object of high interest as it represents a wonderful opportunity of implementing both enantioselectivity 
and photocatalyzed processes. ${ }^{2 c, f 3}$ To this aim, a great deal of work has concerned the development of dual catalytic systems wherein both an organocatalyst and a photoredox catalyst are employed. ${ }^{2 c, f 3}$ Whereas this approach has found a huge success ever since the seminal report of MacMillan et al. was published in $2008,{ }^{4}$ the prospect of using chimeric catalysts which, within the same catalytic entity, encompass an organocatalytic activating group and a photoactive moiety has comparatively been overlooked..$^{5-7}$ Considering visible-light based processes, Bach et al. reported the first chiral organophotocatalyst in which a thioxanthone chromophore and an amide hydrogen bond donor/acceptor were incorporated to a rigid backbone structure (A, Scheme $1(\mathrm{a})){ }^{6 \mathrm{a}-\mathrm{c}}$ It has demonstrated its efficiency in enantioselective [2+2] cycloadditions as well as the deracemization of allenes. Then, the same group also described a thiourea-thioxanthone hybrid photocatalyst which was used to photocyclize a substituted cyclohexanone with a low ee (B, Scheme 1(a)). ${ }^{6 \mathrm{~d}}$ More recently, Alemán et al. designed a bifunctional photoaminocatalyst based on the introduction of a thioxanthone moiety on an imidazolidinone core for the enantioselective $\alpha$-alkylation of aldehydes (C, Scheme 1(a)). ${ }^{6 e}$ In this particular context, given our interest in enantioselective organocatalysis ${ }^{8}$ and photoredox catalysis, ${ }^{9}$ we became interested in designing a new class of photoactive organocatalyst which, based on the BINOL-phosphoric acid backbone, would possess one or two photoactive groups in the $3,3^{\prime}$ position. Such chimeric chemical entities would offer the opportunity, depending on the presence or the absence of an adequate source of light, to either induced a defined asymmetric organocatalytic transformation or, following the concept of assisted tandem catalysis,${ }^{10}$ a photocatalyzed process. Inspired by Bach's and Alemán's works, the heterocyclic thioxanthone unit was selected as the visible light photosactive moety for the synthesis of our targeted bifunctional organo/photo catalysts. ${ }^{3,6,7 c, 11}$ Herein, we describe our initial efforts 
toward the development of this new class of catalysts and their evaluation in the enantioselective multicomponent syntheses of 1,2-diamines (Scheme 1(b)). Worthy of note, during the course of this study, Bach and co-workers reported other $C_{2}$-symmetric chiral photensitizers derived from chiral phosphoric acids which he applied to enantioselective [2+2] photo-cycloadditions. ${ }^{12}$

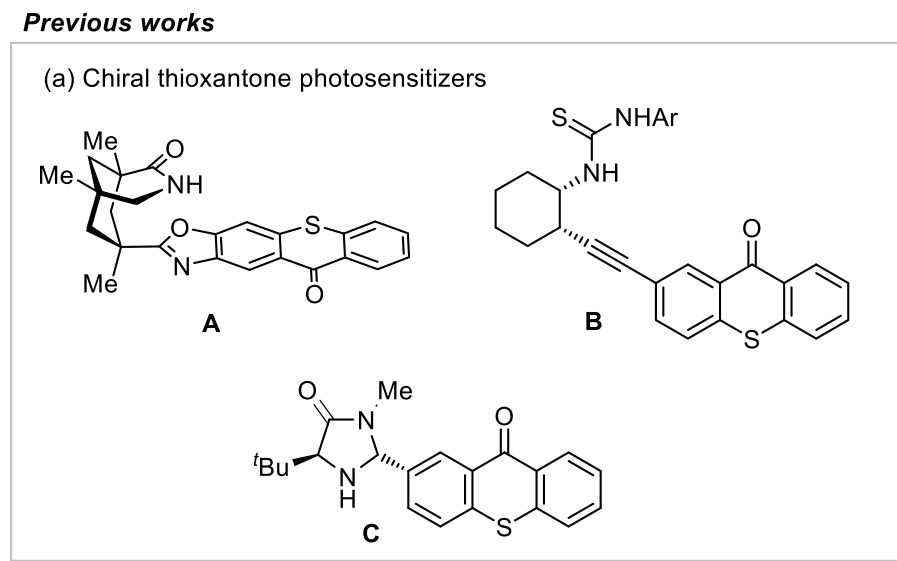

Our works

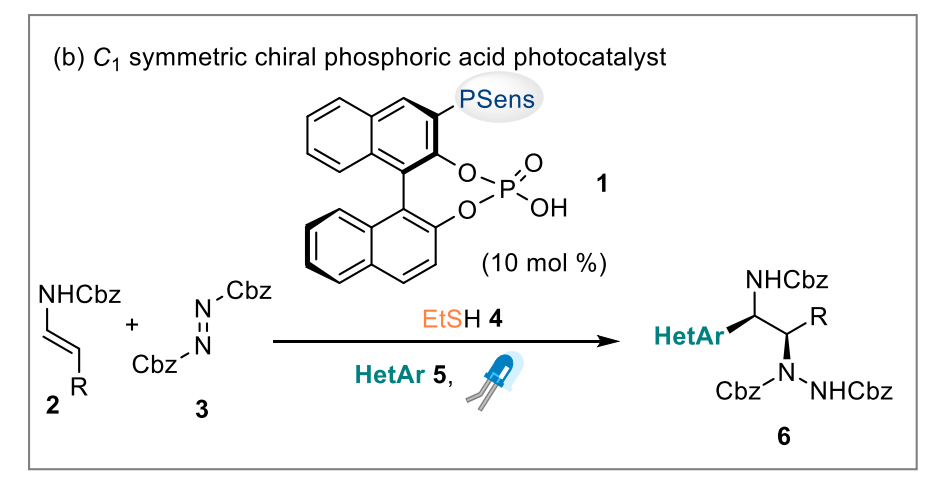

Scheme 1. Enantioselective catalytic photochemical reactions.

\section{RESULTS AND DISCUSSION}

The new phosphoric acids 1a-c bearing a visible-light sensitive thioxanthone moiety were easily accessible in few steps starting from optically pure BINOL derivatives, according to the route outlined in Scheme 2 . The first $C_{2}$-symmetric compound 9 was prepared in a convergent manner by Suzuki-Miyaura cross-coupling of the adequate $(R)$-BINOL dimethyl ether 3,3-bisboronic acid $7^{13}$ 
with the 2-bromo-thioxanthone 8. The second type of $C_{2}$-symmetric MOM-protected BINOL 11 with an alkyne spacer between the BINOL and thioxanthone core was obtained via the Sonogashira coupling of diacetylene (S)-10 with 8. ${ }^{14}$ Additionally, we were interested in synthesizing the $C_{1}$ symmetric catalyst $1 \mathrm{c}$ having a simple thioxanthone unit linked to the $(R)$-BINOL backbone. To this end, starting from 12, the in situ generated mono-substituted BINOL boronic acid ${ }^{15}$ was subjected to Suzuki-Miyaura coupling in the presence of 8 to give the desired product 13 in $45 \%$ yield over twosteps. Finally, the resulting BINOL derivatives 9,11 and 13 were converted into the desired photocatalysts 1a-1c via deprotection and subsequent phosphorylation.

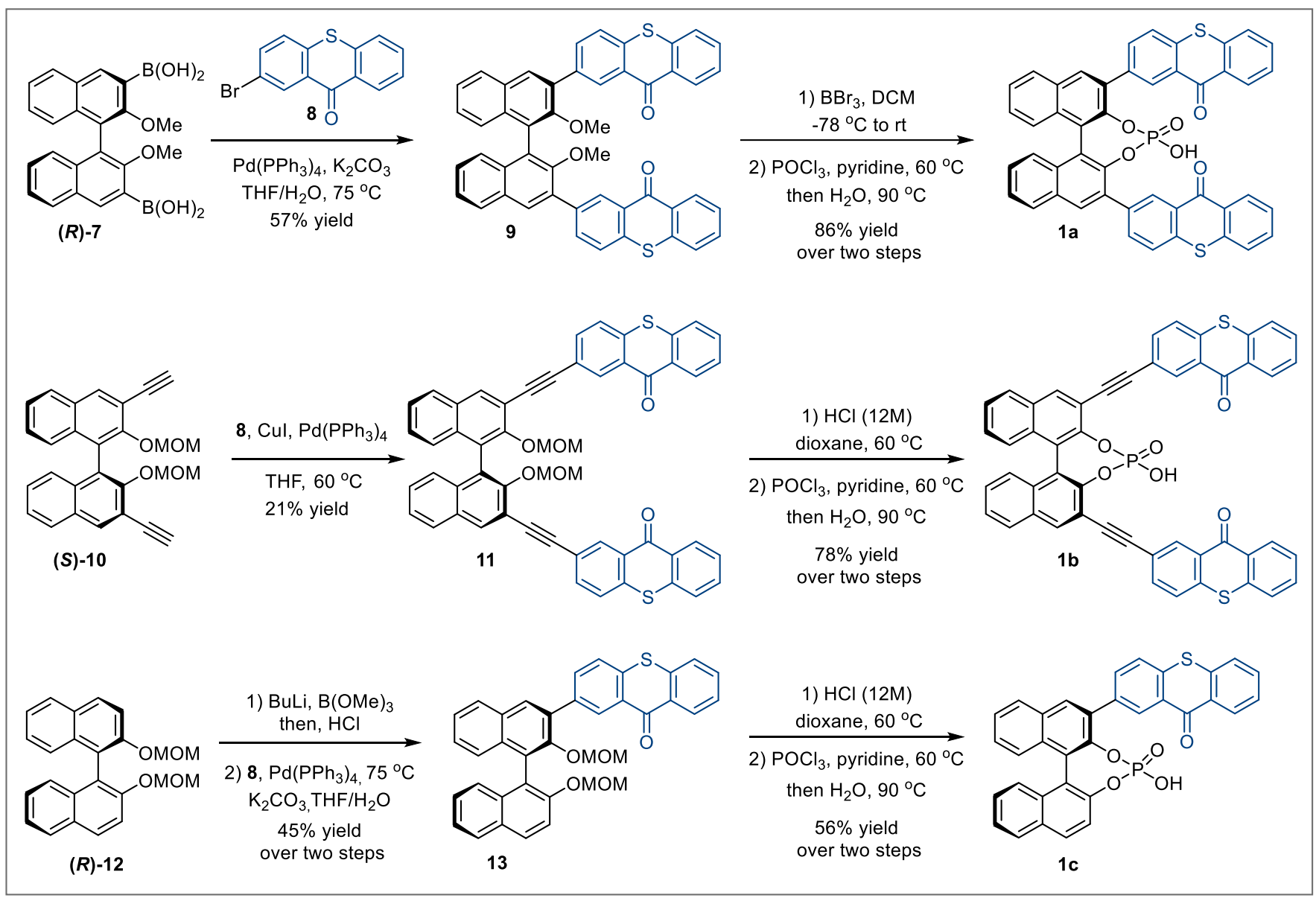

Scheme 2. Syntheses of chiral phosphoric acid photocatalysts. 
With these chimeric organo/photo catalysts 1a-c in hand, we next turned our attention to their photophysical properties by initially recording their UV-visible absorption profiles in DCE. Chiral phosphoric acid-thioxanthone photocatalysts $1 \mathrm{a}, 1 \mathrm{~b}$ and $1 \mathrm{c}$ showed similar absorption properties with $\lambda_{\max }$ at 390, 400 and $390 \mathrm{~nm}$, respectively (Table 1). It confirmed their ability to absorb light in the visible region, until approximately $440 \mathrm{~nm}$. (See the absorption spectra, Figure $\mathrm{S} 1$ in the Supporting Information). The fluorescence emission spectrums of 1a-1c displayed a wide peak centered at 437, 453 and $425 \mathrm{~nm}$, respectively, with moderate fluorescence quantum yields (See the fluorescence spectra, Figure S2 in the Supporting Information). It is interesting to note that $1 \mathrm{a}$ is three times less fluorescent than 1c suggesting an intramolecular bleaching between the two thioxanthone residues. We then determined the electrochemical properties of chiral photocatalysts 1 by conducting cyclic voltammetry (CV) experiments in $\mathrm{DCE}^{16}$ as reported in Table 1 (See the cyclic voltammograms, Figure S3-S5 in the Supporting Information). These derivatives exhibit similar electrochemical behaviors with reversible reduction waves. The reduction peaks of $1 \mathrm{a}, 1 \mathrm{~b}$ and 1c were found at $-1.55,-1.46$ and $-1.53 \mathrm{~V}$ vs SCE, respectively. Then, the redox potential of the excited states $1 \mathrm{a}^{*}-1 \mathrm{c}^{*}$ (range from +1.19 to $+1.24 \mathrm{vs} \mathrm{SCE}$ ) were estimated according to Rehm-Weller approximation. ${ }^{17}$

Table 1. Photophysical and electrochemical data of 1 in DCE.

\begin{tabular}{|c|c|c|c|c|c|c|}
\hline entry & Pcat 1 & $\lambda_{\text {abs }} \mathrm{nm}$ & $\lambda_{\mathrm{em}} \mathrm{nm}$ & $\phi f$ & $\begin{array}{c}\mathrm{E}_{1 / 2}\left(\text { Pcat } 1 / 1^{\bullet-}\right. \\
\mathrm{V} \text { vs SCE })\end{array}$ & $\begin{array}{c}\mathrm{E}^{1 / 2}\left(\text { Pcat } 1^{*} / 1\right. \\
\text { V vs SCE })^{\mathrm{a}}\end{array}$ \\
\hline 1 & $1 \mathbf{a}$ & 390 & 437 & 0.014 & $-1.55 \mathrm{~V}$ & $+1.19 \mathrm{~V}$ \\
\hline 2 & $1 b$ & 400 & 453 & 0.014 & $-1.46 \mathrm{~V}$ & $+1.20 \mathrm{~V}$ \\
\hline 3 & $1 \mathrm{c}$ & 390 & 425 & 0.049 & $-1.53 \mathrm{~V}$ & $+1.24 \mathrm{~V}$ \\
\hline $\begin{array}{l}\text { bsorptio } \\
\text { concent } \\
\text { Pcat } 1 * 1 \\
\text { ibsorptio }\end{array}$ & wavelen & th. $\varepsilon$ molar & $\begin{array}{l}\text { sorption c } \\
\text { a }^{\text {a Calcula }} \\
\text { imated spe }\end{array}$ & $\begin{array}{l}\text { ient. } E^{0} \mathrm{n} \\
\text { ing the } \mathrm{r} \\
\text { opically } \\
\text { nformatic }\end{array}$ & $\begin{array}{l}\text { ured in } 1,2 \text {-dichlor } \\
\text { onship } E^{\circ} \text { ( Pcat } 1^{*} / 1 \\
\text { the position of the }\end{array}$ & $\begin{array}{l}\text { hane }(\mathrm{DCE}) \text { with a } \\
=E^{3 / 2}\left(\mathrm{Pcat} 1 / 1^{\circ-}\right) \\
\text { g wavelength tail }\end{array}$ \\
\hline
\end{tabular}


We then started to explore the catalytic activities of this new catalysts. Last year, we have reported a sequential asymmetric vicinal difunctionalization of $\alpha$-unsubstituted enecarbamates ${ }^{8 c, d}$ by the combination of phosphoric acid and photoredox catalysis. ${ }^{18}$ The sequence, which consists in a three-component electrophilic amination of enecarbamates followed by an indole-based FriedelCrafts reaction, delivers potentially bioactive $\beta$-amino $\alpha$-substituted tryptamine derivatives in excellent enantioselectivity (Scheme 3(a)). The main challenges observed in this "one pot" threecomponent transformation (Scheme 3(b)) was due to competitive processes such as the direct addition of the indole partner to the azodicarboxylate and the polymerization by successive addition of the enecarbamate to the imine intermediate 15 (generated during electrophilic amination). To prevent these competitive reactions, we have developed a strategy based on the use of a thiol as a temporary nucleophile to trap the unstable imine 15 . By means of a subsequent ruthenium-based photocatalytic process, the resulting $\alpha$-carbamoylsulfides 16 allowed the regeneration of the enantioenriched imine intermediates 15 , able so to undergo a very neat Friedel-Crafts reaction with indoles. However, the "one-pot sequential" process was regrettably not feasible because the solvents required for achieving good yields in each step were mismatched (the Ru-catalyzed Friedel-Crafts step was not effective in $\mathrm{CH}_{2} \mathrm{Cl}_{2},{ }^{18,19}$ whereas moderate enantioselectivity/yield were observed in the electrophilic amination carried out in $\mathrm{CH}_{3} \mathrm{CN}$ ). Additionally, it was found that the purification step of 16 was required to provide the $\beta$-amino $\alpha$-substituted tryptamines 17 . On the other hand, when a less nucleophilic pyrazole was used to form the $N$-alkylated product $(\mathrm{R}=\mathrm{Me})$ 6a, moderate enantioselectivity was observed, possibly due to partial racemization of the imine intermediate 15 (Scheme 3(c)). To solve this issue, we hypothesized that our newly developed chiral photocatalyst 
could be good candidates to promote both steps of this sequence (Scheme 3(d)): it would (1) induce high enantioselectivity in the electrophilic amination via the well-organized transition state TS$\mathrm{A} ; ;^{1,8, \mathrm{~d} d}$ (2) promote the photocatalyzed imine regeneration reaction via the $\mathrm{C}-\mathrm{S}$ bonds cleavage of $16^{9-e, 18}$ and (3) allow, while avoiding undesired racemization, the $N$-alkylation step. Indeed, during the second step of the process, the simultaneous dual activation of the two reacting partners 15 and $\mathrm{NuH}$ by the phosphoric acid catalyst was anticipated to accelerate the rate of addition of pyrazoles via TS-B, over racemization (mots probably occurring through imine-enamine tautomerism). To validate these assumptions, we carried out the one pot three-component direct amination of $\alpha$ unsubstituted enecarbamate 2a with dibenzyl azodicarboxylate 3 and ethanethiol 4 in the presence of these new chiral photosensitive phosphoric acid catalysts 1a-c. Based on our previous work, the reaction was initially performed with $10 \mathrm{~mol} \%$ of $\mathrm{C}_{2}$-symmetric 1 a at $-45^{\circ} \mathrm{C}$ in $\mathrm{CH}_{2} \mathrm{Cl}_{2}$, away from the light (Table 2). After $16 \mathrm{~h}$, the pyrazole was added and the resulting reaction mixture was irradiated under blue LED light strips $(7.2 \mathrm{~W}, 420-510 \mathrm{~nm}$, See the emission spectrum, Figure S9 in the Supporting Information) at room temperature under oxygen atmosphere. To our delight, the desired product 6a was isolated in a decent yield (64\%) and with an excellent enantioselectivity (98\% ee) for each diastereomer couple (entry 1$)$. Strikingly, catalyst $1 \mathrm{~b}$ possessing an alkyne linker was found inefficient and only trace amounts of 6 a could be isolated, in nearly racemic form (entry 2). This suggests that both efficiency and selectivity of 1 seem to depend on the exact position of the two active functions (phosphoric acid/thioxanthone). To our delight, the $C_{1}$-symmetric photocatalyst 1c gave better yield (73\% yield) and, contrary to what is generally observed with these types of chiral monophosphoric acid catalysts, without compromising enantioselectivity (entry 3 vs 1 ). ${ }^{20}$ The higher activity of $1 \mathrm{c}$ is very much consistent with the results of quantum yield 
determinations. The use of 3.0 equiv of pyrazole (5a) under standard conditions did increase the yield (entry 4). Interestingly, the reaction promoted by the combined use of two distinct catalysts, the chiral phosphoric acid 14 and thioxanthone, provided the 1,2-diamide derivatives $6 \mathrm{a}$ with a much lower $21 \%$ yield and a significant loss of enantiomeric purity, thus validating the superiority of our bifunctional catalytic strategy (entry 5). Worthy of note, when a similar reaction was performed in absence of thioxanthone, 6a was only detected in trace amounts (entry 6) and, under continuously dark reaction conditions, only the intermediate 16 was formed (entry 7). ${ }^{21}$ Moreover, a parallel control experiments showed that dioxygen (entry 8) was also compulsory for the full sequence to occur (entry 8 ). Finally, a 1-mmol-scale reaction using $1 \mathrm{c}$ was performed, affording the 1,2-diamine product 6a in equal yield and enantioselectivity (entry 9). 


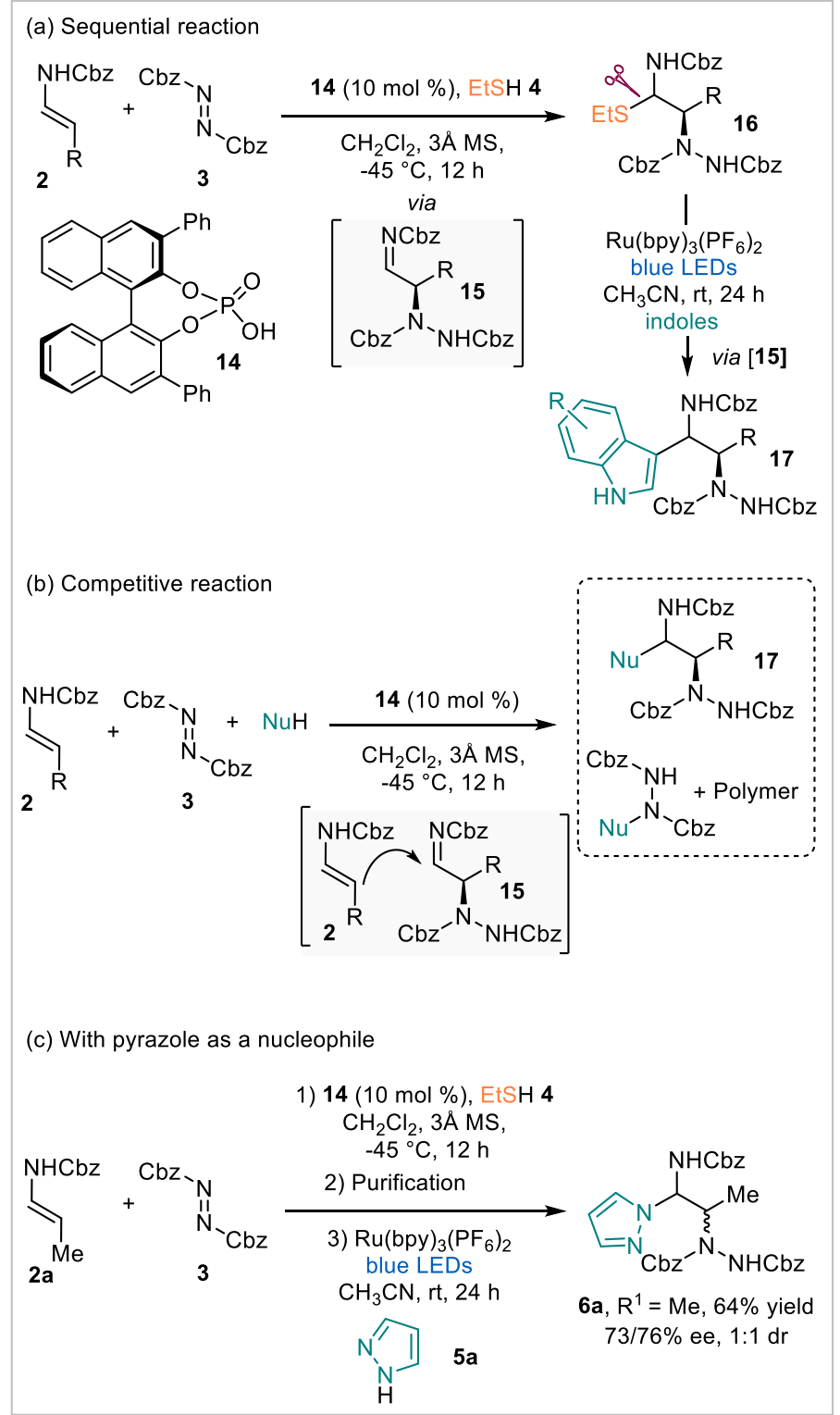

Our proposal

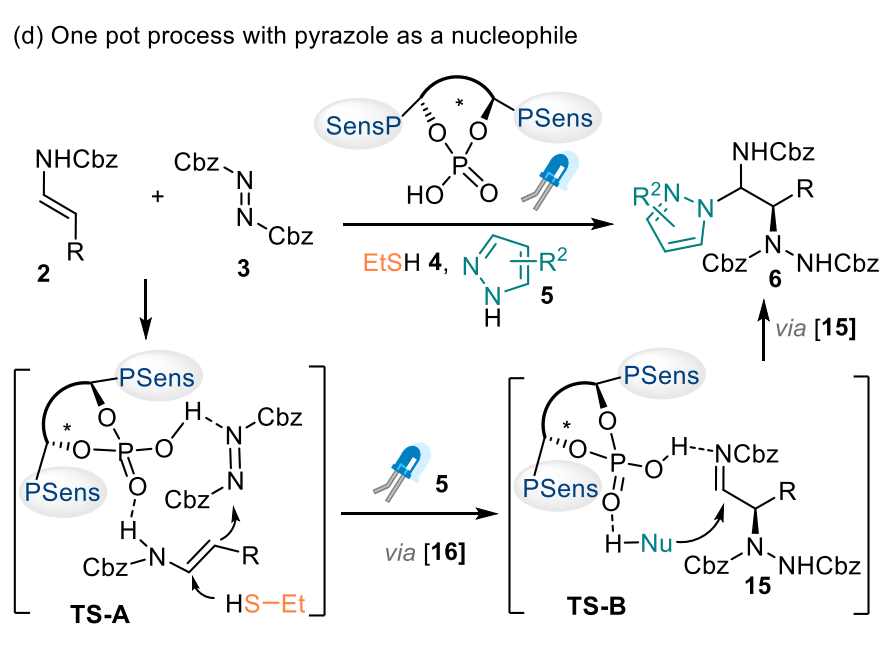

Scheme 3. Asymmetric $\alpha$-amination and photocatalyzed pyrazole addition. 
Table 2. Optimization of the tandem processes.

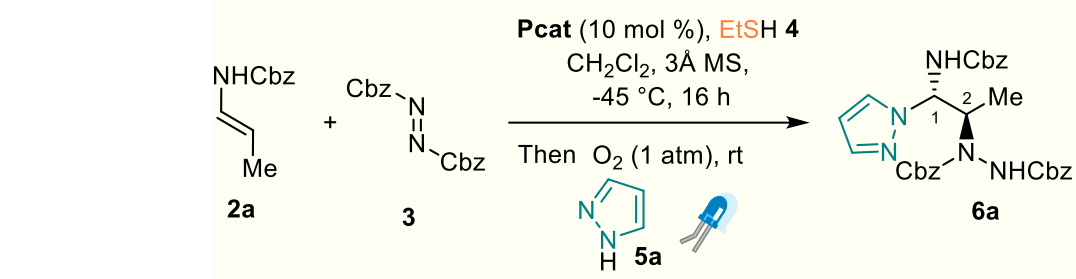

\begin{tabular}{ccccc}
\hline entry & Pcat & yield $(\%)^{\mathrm{a}, b}$ & $d r^{c}$ & $e e(\%)^{d}$ \\
\hline 1 & $\mathbf{1 a}$ & 64 & $6: 4$ & $98 / 98^{e}$ \\
2 & $\mathbf{1 b}$ & 28 & $2: 1$ & $<5$ \\
3 & $\mathbf{1 c}$ & 72 & $7: 3$ & $98 / 98^{e}$ \\
4 & $\mathbf{1 c}^{f}$ & 83 & $7: 3$ & $98 / 97^{e}$ \\
5 & $\mathbf{1 4}^{f, g}$ & 21 & $7: 3$ & $90^{e}$ \\
6 & $\mathbf{1 4}^{f}$ & trace & nd & nd \\
7 & $\mathbf{1 c}^{f, h}$ & trace & nd & nd \\
8 & $\mathbf{1 c}^{f, i}$ & $10^{j}$ & $7: 3$ & nd \\
9 & $\mathbf{1 c}^{f, k}$ & 88 & $7: 3$ & $98 / 97^{e}$ \\
\hline
\end{tabular}

${ }^{a}$ General condition: enecarbamate $1 \mathrm{a}(0.10 \mathrm{mmol})$, dibenzylazodicarboxylate $(0.15 \mathrm{mmol})$, ethanethiol $(0.10 \mathrm{mmol}), 3 \AA$ molecular sieve $(20 \mathrm{mg})$, and $(S)-1(0.010 \mathrm{mmol})$, in $\mathrm{CH}_{2} \mathrm{Cl}_{2}(1.0 \mathrm{~mL})$ at $-45^{\circ} \mathrm{C}$ for $16 \mathrm{~h}$ followed by addition of pyrazole $5 \mathrm{a}(0.15 \mathrm{mmol})$ and irradiation with a blue LED light strips at $\mathrm{rt}$ under $\mathrm{O}_{2}$ atmosphere for $16 \mathrm{~h} .{ }^{b}$ Yields refer to chromatographically pure products. ${ }^{c} d r$ was determined by ${ }^{1} \mathrm{H}$-NMR analysis and anti-diastereoselectivity assumed by analogy with our previous work, see the supporting information. ${ }^{d}$ Enantiomeric excess was determined by HPLC analysis on chiral stationary phase. ${ }^{e}$ Refers to $(2 R)$-absolute configuration assumed by analogy with our previous work, see ref. 8c and 8d. ${ }^{f}$ With $0.30 \mathrm{mmol}$ of pyrazole 5a. ${ }^{g}$ With thioxanthone $(0.020 \mathrm{mmol}) .{ }^{h}$ Reaction fully performed in the dark. ${ }^{i}$ Reaction conducted under argon atmosphere. ${ }^{j}$ Determined by ${ }^{1} \mathrm{H}$-NMR using bromoform as internal standard. ${ }^{k}$ Reaction performed on a $1.0 \mathrm{mmol}$ scale of $2 \mathrm{a}$ and $490 \mathrm{mg}$ of $6 \mathbf{a}$ was obtained.

Having established the optimal reaction conditions, we then probed the generality of this catalytic asymmetric $\beta$-amination/nucleophilic addition sequence with a variety of azoles and enecarbamates. As shown in Table 3, a range of pyrazoles bearing electron-donating or electronwithdrawing group at various positions on the heteroaromatic ring proved compatible and smoothly afforded the desired 1,2-diamide derivatives $6 \mathrm{~b}-6 \mathrm{~d}$ in good yields and excellent enantioselectivities, although with somewhat moderate diastereoselectivities. The reaction of benzopyrazole afforded 
the corresponding adduct $6 \mathrm{e}$ in a high enantioselectivity and 3:1 dr. In addition, enecarbamates $\beta$ substituted with linear alkyl chains reacted efficiently to produce the desired products $6 \mathrm{f}-6 \mathbf{j}$ with high enantioselectivities. The presence of a tert-butyldiphenylsilyl ether was well tolerated, which allowed to obtain the corresponding enantioenriched products $(6 \mathrm{~m}-\mathrm{o})$. The introduction of isopropyl group at the $\beta$-position of the ene moiety gave $6 \mathbf{k}$ in reduced yield. However, when the $\beta$ substituted cyclopropyl enecarbamate was used, the expected 1,2-diamine 61 was isolated in higher yield. We also tested the enantioselective tandem three-component electrophilic amination of enecarbamates with other nucleophiles than azoles. While the reaction with trimethoxybenzene gave only trace of product, 7-bromoindole reacted to form the desired product $6 \mathrm{p}$ with an excellent enantioselectivity and as a single diastereomer.

Table 3. Substrate scope of the enantioselective synthesis of 1,2-diamines. ${ }^{a-d}$ 


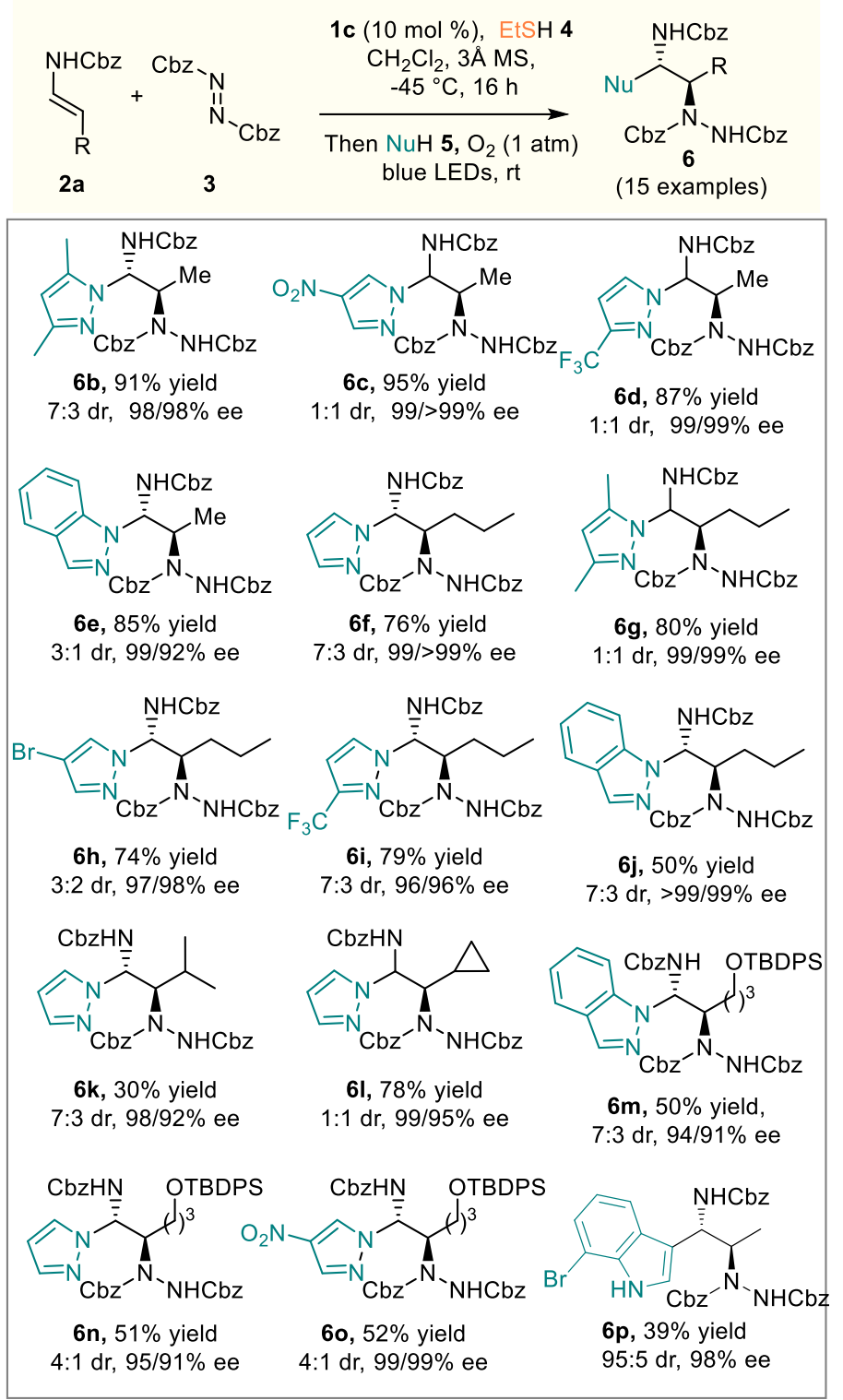

${ }^{a}$ General condition: enecarbamate $1(0.10 \mathrm{mmol})$, dibenzylazodicarboxylate $(0.15 \mathrm{mmol})$, ethanethiol $(0.10 \mathrm{mmol}), 3 \AA$ molecular sieve $(20 \mathrm{mg})$, and $(S)-1 \mathrm{c}(0.010 \mathrm{mmol})$ in $\mathrm{CH}_{2} \mathrm{Cl}_{2}(1.0 \mathrm{~mL})$ at $-45{ }^{\circ} \mathrm{C}$ for $16 \mathrm{~h}$ followed by addition of $5(0.30$ mmol) with a blue LED light strips at rt under $\mathrm{O}_{2}$ atmosphere for $16 \mathrm{~h} .{ }^{b}$ Yields refer to chromatographically pure products. ${ }^{c}$ dr determined by ${ }^{1} \mathrm{H}-\mathrm{NMR}$ analysis. ${ }^{d}$ Enantiomeric excess was determined by HPLC analysis on chiral stationary phase.

Based on other reports ${ }^{2,6,7 c, 11,22}$ and our previous studies, ${ }^{9 b-e, 18}$ we proposed a plausible reaction pathway for the photochemical C-S bonds cleavage as depicted in Scheme 4. After the electrophilic three-component thio-amination step, the resulting chiral $\alpha$-carbamoylsulfide 16 would be oxidized by the excited chiral thioxanthone $1 c^{*}, 2 e, 2 \mathrm{~h}, 6,7 \mathrm{c}, 11,23$ thereby generating the ketyl radical anion 17 and the sulfur radical cation 19. A single electron transfer from the ketyl radical anion 17 to $\mathrm{O}_{2}$ would 
regenerate the thioxanthone photocatalyst $1 \mathrm{c}$ and simultaneously produce the superoxide radical anion $\left(\mathrm{O}_{2}^{-}\right)$18. In the meantime, generation of a positive charge on $\mathrm{S}$ in the radical cation 19 would weaken the C-S bond resulting in a mesolytic cleavage to produce the imine 15 and the thiyl radical 20 (the later would dimerize to form the disulfide 21). Finally, the addition of the azole partner 5 onto the imine 15 and the subsequent hydrogen atom abstraction from $\mathrm{O}_{2}{ }^{-}$, would afford the enantioenriched diamine product $6 .{ }^{24}$ Interestingly, the conservation of enantioselectivity which is typically observed supports a dual activation mechanism in which the phosphoric acid 1c would ensure a rapid attack of the azole 5 onto the imine 15 via the dual activation of both reaction partners according to the presumed hydrogen bonding TS-B (Scheme 3). The observations provided by the control experiments reported above (Table 2, entries 6 to 8) established that visible light, thioxanthone and oxygen were all essential which is in good agreement with the proposed mechanism. The fact that the reaction proceeded in merely $10 \%$ conversion in the absence of $\mathrm{O}_{2}$ (entry 8) also suggests that no turnover was achievable without it. Hence, $\mathrm{O}_{2}$ might be involved in the oxidative regeneration of the catalyst, the fast dimerization of the thiyl radical $\mathbf{2 0}$ disfavoring a classical radical propagation process. The on/off visible light irradiation experiment supports a nonradical-chain propagation process (see Table S2 and Figure S10, in the Supporting information). ${ }^{25}$ However, another pathway involving the oxidation of the carbamoylsulfide 16 by singlet oxygen (generated by triplet-triplet annihilation between natural triplet oxygen and the triplet excited state of $1 \mathrm{c})^{7 \mathrm{c}}$ could also explain the formation of the sulfur radical cation 19 (Path 2). To distinguish between these two mechanisms, the C-S bond cleavage of 16 was carried out in the presence of the singlet oxygen quencher, DABCO, and the superoxide radical anion quencher, TEMPO (Scheme 5). In both cases, the desired product was isolated in substantial amount (Scheme 5, 52 and 58\%, 
respectively) but in lower yield when compared to standard condition (entry 4, Table 2). Thus, these results do not allow us to clearly discriminate between these two possible oxidation mechanisms. ${ }^{26}$ Moreover, when we conducted fluorescence quenching experiments of $1 \mathrm{c}^{*}$, a nonlinear Stern-Volmer relationship between Io/I and the concentrations of $16 \mathrm{a}$ was observed (Figure S7 and S8 in the Supporting Information). If the quenching mechanism is mainly due to a dynamic process or a static process, a linear Stern-Volmer relationship is expected. In our case, we observed a higher fluorescence intensity with increased concentrations of 16a, suggesting the formation of a supramolecular complex between the photocatalyst $1 \mathrm{c}$ and compound $16 \mathrm{a}$ that would enhance the fluorescence of 1c. Accordingly, this prevents us from quantifying the efficiency of the photoinduced electron transfer in this system through Stern-Volmer analysis and thus we cannot conclude about the mechanism involved. Finally, the electrochemical oxidation potential of the $\alpha$ carbamoylsulfide $16 \mathrm{a}\left(\mathrm{E}_{\mathrm{P} / 2}=+1.56 \mathrm{~V}\right.$ vs SCE, Figure S6 in the Supporting Information) and that of the photoexcited state of the chiral thioxanthone $1 \mathrm{c}^{*}\left(\mathrm{E}^{\mathrm{T} / 2}=+1.24 \mathrm{~V} \text { vs. SCE }\right)^{27}$ suggest that a direct electron transfer is not thermodynamically favorable. Hence, it advocates that such a process most probably proceeds via the second pathway (Scheme 4, path 2). ${ }^{28}$ 


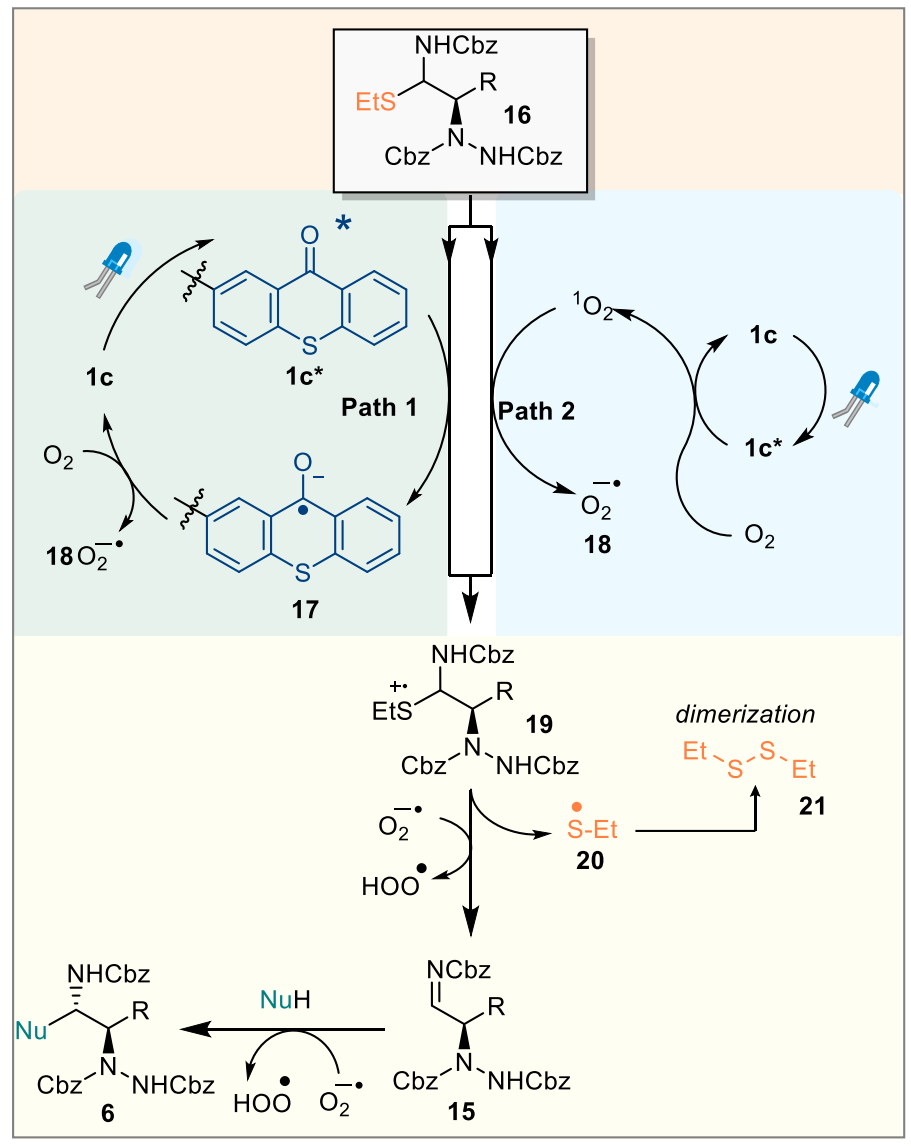

Scheme 4. Considered reaction mechanisms

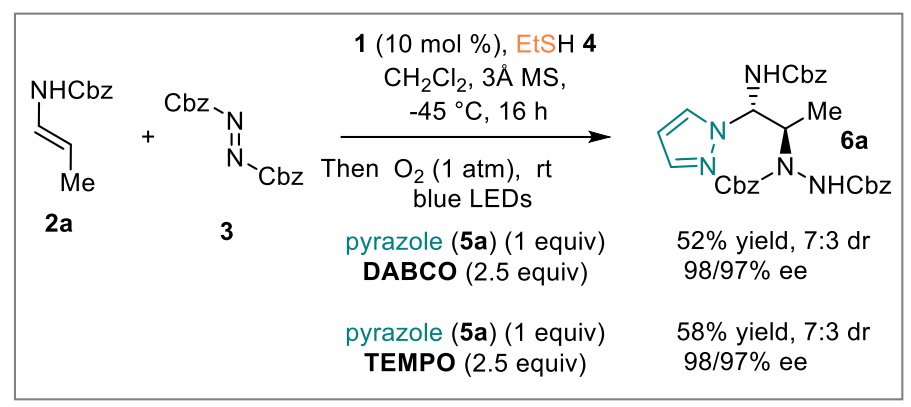

Scheme 5. Control experiments

\section{CONCLUSION}

In summary, we have developed a new family of chimeric chiral photocatalysts in which a BINOL-derived phosphoric acid embeds one or two thioxanthone dyes. We have demonstrated the efficiency of these new photocatalysts in a one pot three-component electrophilic amination 
reaction followed by a photoinduced a nucleophilic addition of various azoles. This assisted tandem process gives access to fully substituted 1,2-diamines in excellent enantioselectivities. Notably, the monosubstituted $C_{1}$-symmetric organo/photo catalyst exhibits a superior catalytic efficiency than the $C_{2}$-symmetric counterparts. Further studies and applications of these novel chiral catalysts are currently underway.

\section{EXPERIMENTAL SECTION}

Materials and General methods. All reactions requiring anhydrous conditions and inert atmosphere were carried out under argon atmosphere in dried glassware. Immersion coolers IMC-40 and TC100E fitted with a temperature probe and a flexible cooling tube were used to perform reactions at low temperature. The heat source employed for the synthesis of catalyst and substrates was an oil bath. Solvents were distilled by standard methods using the appropriate drying agent and stored over molecular sieves under argon. All other reagents were obtained from commercial suppliers and directly used unless otherwise noted. Flash column chromatography was carried out using 40-63 $\mu \mathrm{m}$ particle sized silica gel with air pressure. Analytical thin layer chromatography (TLC) plates (silica gel 60 F254) were visualized either with a UV lamp $(254 \mathrm{~nm})$, or by submersion in potassium permanganate, ninhydrine or iodine. Melting points were recorded using a melting point apparatus (Büchi B-540) and are uncorrected. Specific rotations for chiral compounds were recorded on a Perkin Elmer 141 using sodium D ray $(589 \mathrm{~nm})$ at room temperature in a $700-\mu \mathrm{L}$ cell with a path length of $1 \mathrm{dm}$ in $\mathrm{CH}_{2} \mathrm{Cl}_{2}$. Infrared spectra were recorded on a Perkin Elmer Spectrum BX FT-IR spectrometer and the characteristic IR absorption frequencies are reported in $\mathrm{cm}^{-1}$. Proton NMR $\left({ }^{1} \mathrm{H}\right)$ spectra were recorded on a Bruker Avance $500 \mathrm{MHz}$ or $300 \mathrm{MHz}$ and proton-decoupled carbon $13 \mathrm{NMR}\left({ }^{13} \mathrm{C}\left\{{ }^{1} \mathrm{H}\right\}\right)$ spectra were recorded at $75 \mathrm{MHz}$. NMR experiments were carried out in deuterochloroform $\left(\mathrm{CDCl}_{3}\right)$ or in 
trideuteroacetonitrile $\left(\mathrm{CD}_{3} \mathrm{CN}\right)$. Proton-decoupled fluorine ${ }^{19} \mathrm{~F}$ NMR $\left({ }^{19} \mathrm{~F}\left\{{ }^{1} \mathrm{H}\right\}\right)$ spectra were recorded at $282 \mathrm{MHz}$. Proton-decoupled phosphorus ${ }^{31} \mathrm{P}$ NMR $\left({ }^{31} \mathrm{P}\left\{{ }^{1} \mathrm{H}\right\}\right)$ spectra were recorded at $202 \mathrm{MHz}$. Chemical shifts $(\delta)$ are reported in parts per million (ppm) relative to residual solvent as an internal reference $\left({ }^{1} \mathrm{H}: \quad 7.26,{ }^{13} \mathrm{C}: 77.16 \mathrm{ppm}\right.$ for $\mathrm{CHCl}_{3} / \mathrm{CDCl}_{3}$ and ${ }^{1} \mathrm{H}: 1.94,{ }^{13} \mathrm{C}: 1.32 \mathrm{ppm}$ for $\mathrm{CHD}_{2} \mathrm{CN} / \mathrm{CD}_{3} \mathrm{CN}$ ). The following abbreviations are used for the multiplicities: s: singlet; d: doublet; t: triplet; q: quadruplet; quint: quintuplet; m: multiplet or overlap of non-equivalent resonances; br s: broad singlet; app: apparent; rot: rotamer. Coupling constants $(J)$ are reported in Hertz $(\mathrm{Hz})$. High resolution mass spectra were determined on an AEI MS-9 using electrospray ionization (ESI) and a time-of-flight (TOF) analyzer. Enantiomeric excesses were determined by high performance liquid chromatography (HPLC) equipped with diode array UV detectors, using Chiralpak ${ }^{\circledR}$ AD-H, OD-H, AS$\mathrm{H}, \mathrm{OJ}-\mathrm{H}, \mathrm{IA}$ and IB columns. Reactions were irradiated using a ruban of Flexled INSPIRE LED lamp (45 LEDs, $7.2 \mathrm{~W}, \lambda_{\max }=458 \mathrm{~nm}$; commercial supplier: Linda Zhu Yichun Yaozhou Electronic Technology Co; purchased from Leroy Merlin) without any filters, and a cooling fan was placed over the reaction vessels to keep temperature at $25^{\circ} \mathrm{C}$. The spectral distribution and intensity of the lamp is shown in Figure S9 (in the Supporting Information). The distance from the light source to the irradiation vessel is $5 \mathrm{~cm}$. The round bottom flask was made of borosilicate glass. Enecarbamate starting materials $\mathbf{2 a - 2 e}$ were prepared according to literature procedure. ${ }^{29}$

\section{Preparation of Photocatalyst (1a):}

(R)-2,2'-(2,2'-dimethoxy-[1,1'-binaphthalene]-3,3'-diyl)bis(9H-thioxanthen-9-one) (9). In an 100 $\mathrm{mL}$ round bottom flask equipped with a stir bar and a reflux condenser were placed $\mathbf{7}^{\mathbf{1 3}}$ (1 equiv, 2.0 mmol, $0.8 \mathrm{~g}$ ), 2-bromo-thioxanthone $\mathbf{8}^{14}$ (2.3 equiv, $\left.4.6 \mathrm{mmol}, 1.33 \mathrm{~g}\right), \mathrm{K}_{2} \mathrm{CO}_{3}$ (6 equiv, $12 \mathrm{mmol}, 1.7$ g) and $\mathrm{Pd}\left(\mathrm{PPh}_{3}\right)_{4}(0.1$ equiv, $0.01 \mathrm{mmol}, 0.23 \mathrm{~g})$ under argon. Then, a degassed 10:1 mixture of $\mathrm{THF} / \mathrm{H}_{2} \mathrm{O}$ was added through the condenser $(56 \mathrm{~mL})$. The resulting mixture was vigorously stirred and refluxed for $12 \mathrm{~h}$ under an argon atmosphere. The mixture was cooled to room temperature, diluted with 
brine and $\mathrm{CH}_{2} \mathrm{Cl}_{2}(50 \mathrm{~mL})$ and transferred into a separatory funnel. The layers were separated. The aqueous layer was extracted with $\mathrm{CH}_{2} \mathrm{Cl}_{2}(3 \times 50 \mathrm{~mL})$. The combined organic extracts were washed with brine, dried over $\mathrm{MgSO}_{4}$, filtered and concentrated under reduced pressure. The residue was purified by flash column chromatography on silica gel using Petroleum Ether (PE)/EtOAc as eluent (gradient from 10:1 to 5:1) to afford 9 as a yellow solid $(859 \mathrm{mg}, 1.1 \mathrm{mmol}, 57 \%)$. $[\alpha]_{\mathrm{D}}{ }^{25}-75.0(c 1.0$, $\mathrm{CH}_{2} \mathrm{Cl}_{2}$ ); IR (neat) $v\left(\mathrm{~cm}^{-1}\right): 1736,1636,1591,1493,1475,1457,1437,1405,1388,1353,1321,1283$, $1264,1246,1213,1163,1148,1122,1069,1040,1017,998,960,928,892 ; \mathrm{mp}: 102-109{ }^{\circ} \mathrm{C} ;{ }^{1} \mathrm{H}\left\{{ }^{13} \mathrm{C}\right\}$ NMR $\left(300 \mathrm{MHz}, \mathrm{CDCl}_{3}\right) \delta 9.03(\mathrm{~d}, J=0.9 \mathrm{~Hz}, 2 \mathrm{H}), 8.68(\mathrm{~d}, J=8.0 \mathrm{~Hz}, 2 \mathrm{H}), 8.19-8.08(\mathrm{~m}, 4 \mathrm{H}), 7.97$ $(\mathrm{d}, J=8.1 \mathrm{~Hz}, 2 \mathrm{H}), 7.71-7.57(\mathrm{~m}, 6 \mathrm{H}), 7.54-7.41(\mathrm{~m}, 4 \mathrm{H}), 7.37-7.23(\mathrm{~m}, 3 \mathrm{H}), 3.23(\mathrm{~s}, 6 \mathrm{H})$ ${ }^{13} \mathrm{C}\{1 \mathrm{H}\} \mathrm{NMR}\left(75 \mathrm{MHz}, \mathrm{CDCl}_{3}\right) \delta 180.0,153.9,137.4,137.2,136.4,134.0,133.8,133.5,132.3,131.0$ 130.9, 130.0, 129.9, 129.5, 129.3, 128.3, 126.8, 126.4, 126.1, 125.9, 125.8, 125.3, 60.8; HRMS (ESI/QTOF) m/z: $[\mathrm{M}+\mathrm{H}]^{+}$Calcd for $\mathrm{C}_{48} \mathrm{H}_{31} \mathrm{O}_{4} \mathrm{~S}_{2}[\mathrm{M}+\mathrm{H}]^{+}$735.1683, Found 735.1694.

\section{(R)-2,2'-((4R)-4-hydroxy-4-oxidodinaphtho[2,1-d:1',2'-f][1,3,2]dioxaphosphepine-2,6-diyl)bis(9H-}

thioxanthen-9-one) (1a). 9 (1 equiv, $1.24 \mathrm{mmol}, 0.91 \mathrm{~g}$ ) was dissolved in dry $\mathrm{CH}_{2} \mathrm{Cl}_{2}(24 \mathrm{~mL})$ in a 50 $\mathrm{mL}$ dry round bottom flask covered by aluminum foil and cooled at $-78{ }^{\circ} \mathrm{C} . \mathrm{BBr}_{3}(3$ equiv, $1 \mathrm{M}$ in $\mathrm{CH}_{2} \mathrm{Cl}_{2}, 3.7 \mathrm{mmol}, 3.7 \mathrm{~mL}$ ) was added slowly. After complete addition, the mixture was stirred for 30 min at $-78{ }^{\circ} \mathrm{C}$ and for $4 \mathrm{~h}$ at room temperature. Upon completion (monitored by TLC), the mixture was cooled to $0{ }^{\circ} \mathrm{C}$ and carefully quenched with water $(20 \mathrm{~mL})$. The aqueous layer was extracted with $\mathrm{CH}_{2} \mathrm{Cl}_{2}(3 \times 30 \mathrm{~mL})$. The combined organic phases were concentrated under reduced pressure. The residue $(0.88 \mathrm{~g})$ was weighed in a $2.5 \mathrm{~mL}$ dry round bottom flask. Pyridine $(0.2 \mathrm{~mL})$ and freshly distilled $\mathrm{POCl}_{3}(2.0$ equiv, $2.48 \mathrm{mmol}, 0.23 \mathrm{~mL})$ were added at room temperature under argon. The mixture was stirred for $12 \mathrm{~h}$ at $60{ }^{\circ} \mathrm{C}$. Upon completion (monitored by TLC), the mixture was cooled to room temperature, water $(0.25 \mathrm{~mL})$ was added and the reaction mixture was stirred under reflux for $3 \mathrm{~h}$. Then aq. $\mathrm{HCl}(6 \mathrm{M}, 1 \mathrm{~mL})$ was added. The aqueous phase was extracted with $\mathrm{CH}_{2} \mathrm{Cl}_{2}(3 \times 20 \mathrm{~mL})$. The combined organic phases were concentrated under reduced pressure, and the residue was purified by 
flash column chromatography on silica gel using $\mathrm{CH}_{2} \mathrm{Cl}_{2} / \mathrm{MeOH}$ as eluent (10:1). The obtained solid was then taken up in a mixture of aq. $\mathrm{HCl}(6 \mathrm{M}) /$ dioxane $(1: 1,30 \mathrm{~mL})$ and stirred at $60{ }^{\circ} \mathrm{C}$ until complete solubilization. The mixture was then extracted with $\mathrm{CH}_{2} \mathrm{Cl}_{2}(3 \times 20 \mathrm{~mL})$. The combined organic extracts were concentrated under vacuum to afford $(\boldsymbol{R})-\mathbf{1 a}$ as a yellow solid $(0.82 \mathrm{~g}, 1.1 \mathrm{mmol}, 86 \%)$. $[\alpha]_{\mathrm{D}}{ }^{25}$ $+176.0\left(c\right.$ 1.0, $\left.\mathrm{CH}_{2} \mathrm{Cl}_{2}\right)$; IR (neat) $\vee\left(\mathrm{cm}^{-1}\right): 1633,1589,1496,1477,1460,1438,1420,1391,1318$, 1263, 1186, 1078, 1018, 947, 883, 864, 825; mp: 326-338 ${ }^{\circ} \mathrm{C} ;{ }^{1} \mathrm{H}\left\{{ }^{13} \mathrm{C}\right\}$ NMR $\left(300 \mathrm{MHz}, \mathrm{DMSO}-d_{6}\right) \delta$ $8.79(\mathrm{~s}, 2 \mathrm{H}), 8.68-8.61(\mathrm{~m}, 2 \mathrm{H}), 8.52(\mathrm{~d}, J=7.9 \mathrm{~Hz}, 2 \mathrm{H}), 8.27(\mathrm{~s}, 2 \mathrm{H}), 8.16(\mathrm{~d}, J=8.2 \mathrm{~Hz}, 2 \mathrm{H}), 7.93$ $(\mathrm{dd}, J=16.9,8.2 \mathrm{~Hz}, 4 \mathrm{H}), 7.81(\mathrm{t}, J=7.5 \mathrm{~Hz}, 2 \mathrm{H}), 7.61(\mathrm{t}, J=7.5 \mathrm{~Hz}, 2 \mathrm{H}), 7.51(\mathrm{t}, J=7.5 \mathrm{~Hz}, 2 \mathrm{H})$, $7.35(\mathrm{t}, J=7.6 \mathrm{~Hz}, 2 \mathrm{H}), 7.19(\mathrm{~d}, J=8.6 \mathrm{~Hz}, 2 \mathrm{H}) .{ }^{13} \mathrm{C}\left\{{ }^{1} \mathrm{H}\right\} \mathrm{NMR}\left(75 \mathrm{MHz}, \mathrm{DMSO}-d_{6}\right) \delta 178.9,151.3$ $137.4,136.5,135.2,134.4,133.9,133.0,130.2,129.8,129.1,128.7,128.4,128.3,128.2,126.8,126.6$, 126.5, 126.2, 124.0, 123.1, 115.0. ${ }^{31} \mathrm{P}\left\{{ }^{1} \mathrm{H}\right\} \quad$ NMR (202 MHz, DMSO- $\left.d_{6}\right) \delta 3.43$; HRMS (ESI/Q-TOF) m/z: $[\mathrm{M}-\mathrm{H}]^{-}$Calcd for $\mathrm{C}_{46} \mathrm{H}_{24} \mathrm{O}_{6} \mathrm{PS}_{2}$ 767.0752; Found 767.0758.

\section{Preparation of Photocatalyst (1b):}

\section{(S)-2,2' -((2,2'-bis(methoxymethoxy)-[1,1'-binaphthalene]-3,3'-diyl)bis(ethyne-2,1-diyl))bis(9H-}

thioxanthen-9-one) (11). A $250 \mathrm{~mL}$ round bottom flask was charged with $(\boldsymbol{S})$-10 (1 equiv, 2 mmol, $0.84 \mathrm{~g})$, 2-bromo-thioxanthone $\mathbf{8}^{14}$ (2.5 equiv, $\left.5.21 \mathrm{mmol}, 1.45 \mathrm{~g}\right), \mathrm{Pd}\left(\mathrm{PPh}_{3}\right)_{4}(0.1$ equiv, $0.2 \mathrm{mmol}, 0.23$ g), CuI (0.2 equiv, $0.4 \mathrm{mmol}, 0.076 \mathrm{~g})$ and purged with argon. A mixture of freshly distilled and degassed $\mathrm{Et}_{3} \mathrm{~N} / \mathrm{THF}(1: 1,100 \mathrm{~mL})$ was added and the reaction was stirred for $8 \mathrm{~h}$ at $60{ }^{\circ} \mathrm{C}$. After cooling down to room temperature, diethyl ether $(50 \mathrm{~mL})$ was added. The heterogeneous mixture was filtered through a pad of Celite, washing with diethyl ether $(50 \mathrm{~mL})$. The filtrate was concentrated in vacuum, and the resulting residue was purified by flash column chromatography on silica gel using PE/EtOAc as eluent (gradient from 10:1 to $5: 1)$ to give $(\boldsymbol{S})-\mathbf{1 1}$ as a yellow solid (859 mg, $1.10 \mathrm{mmol}, 21 \%)$. $[\alpha]_{\mathrm{D}}{ }^{25}$ $-44\left(c\right.$ 1.0, $\left.\mathrm{CH}_{2} \mathrm{Cl}_{2}\right)$; IR (neat) $v\left(\mathrm{~cm}^{-1}\right): 1638,1589,1470,1437,1396,1317,1283,1264,1226,1202$ 1155, 1119, 1096, 1076, 1056, 1033, 984, 968, 911, 892; mp: 137-164 ${ }^{\circ} \mathrm{C} ;{ }^{1} \mathrm{H}\left\{{ }^{13} \mathrm{C}\right\} \mathrm{NMR}(300 \mathrm{MHz}$ 
$\left.\mathrm{CDCl}_{3}\right) \delta 8.80(\mathrm{~d}, J=1.5 \mathrm{~Hz}, 2 \mathrm{H}), 8.62(\mathrm{~d}, J=7.9 \mathrm{~Hz}, 2 \mathrm{H}), 8.29(\mathrm{~s}, 2 \mathrm{H}), 7.90(\mathrm{~d}, J=8.1 \mathrm{~Hz}, 2 \mathrm{H}), 7.75$

$(\mathrm{dd}, J=8.4,1.7 \mathrm{~Hz}, 2 \mathrm{H}), 7.67-7.41(\mathrm{~m}, 10 \mathrm{H}), 7.33(\mathrm{dd}, J=13.9,7.4 \mathrm{~Hz}, 4 \mathrm{H}), 5.24(\mathrm{~d}, J=6.1 \mathrm{~Hz}, 2 \mathrm{H})$,

$5.01(\mathrm{~d}, J=6.1 \mathrm{~Hz}, 2 \mathrm{H}), 2.58(\mathrm{~s}, 6 \mathrm{H}) ;{ }^{13} \mathrm{C}\{1 \mathrm{H}\} \mathrm{NMR}\left(75 \mathrm{MHz}, \mathrm{CDCl}_{3}\right) \delta 179.2,153.1,137.3,136.8$, $134.52,134.48,134.0,132.9,132.5,130.4,130.0,129.2,129.1,127.7,127.5,126.6$ (2 peaks overlapping), 126.2, 126.1, 125.9, 125.7, 121.5, 116.9, 99.0, 92.6, 87.9, 56.2; HRMS (ESI/Q-TOF) $\mathrm{m} / \mathrm{z}:[\mathrm{M}+\mathrm{Na}]^{+}$Calcd for $\mathrm{C}_{54} \mathrm{H}_{34} \mathrm{O}_{6} \mathrm{~S}_{2} \mathrm{Na}$ 865.1695; Found 865.1702.

\section{(S)-2,2'-((2,2'-bis(methoxymethoxy)-[1,1'-binaphthalene]-3,3'-diyl)bis(ethyne-2,1-diyl))bis(9H-}

thioxanthen-9-one) (22). Hydrochloric acid (24 equiv, 12M in methanol, $1.5 \mathrm{mmol}, 0.12 \mathrm{~mL}$ ) was added to a solution of $(S)-11$ (1 equiv, $0.06 \mathrm{mmol}, 51 \mathrm{mg})$ in dioxane $(2 \mathrm{~mL})$ and heated at $60{ }^{\circ} \mathrm{C}$ for 12 h. After cooling down to room temperature, water $(5 \mathrm{~mL})$ and $\mathrm{CH}_{2} \mathrm{Cl}_{2}(5 \mathrm{~mL})$ were added. The phases were separated and the organic phase was extracted with $\mathrm{CH}_{2} \mathrm{Cl}_{2}(2 \times 5 \mathrm{~mL})$. The combined organic phases were washed with aq. $\mathrm{NaHCO}_{3}(10 \mathrm{~mL})$, dried over $\mathrm{MgSO}_{4}$, filtered and concentrated under reduced pressure. The residue was purified by flash column chromatography on silica gel using PE/EtOAc as eluent (gradient 5:1 to $1: 1)$ to afford $(\boldsymbol{S})-22$ as a yellow solid $(0.041 \mathrm{~g}, 0.057 \mathrm{mmol}, 95 \%)$ which was used in the next step without further purification. $[\alpha]_{\mathrm{D}}{ }^{25}-60\left(c\right.$ 1.0, $\left.\mathrm{CH}_{2} \mathrm{Cl}_{2}\right)$; IR (neat) $v$ $\left(\mathrm{cm}^{-1}\right): 1639,1588,1575,1470,1436,1396,1319,1286,1265,1190,1146,1120,1074,1019,967,937$, 915, 887, 873; mp: $188-227{ }^{\circ} \mathrm{C} ;{ }^{1} \mathrm{H}\left\{{ }^{13} \mathrm{C}\right\}$ NMR (300 MHz, DMSO- $\left.d_{6}\right) \delta 9.14(\mathrm{~s}, 2 \mathrm{H}), 8.69$ (s, $\left.2 \mathrm{H}\right), 8.47$ $(\mathrm{d}, J=10.4 \mathrm{~Hz}, 2 \mathrm{H}), 8.35(\mathrm{~s}, 2 \mathrm{H}), 8.00-7.92(\mathrm{~m}, 5 \mathrm{H}), 7.89-7.87(\mathrm{~m}, 2 \mathrm{H}), 7.83-7.82(\mathrm{~m}, 2 \mathrm{H}), 7.65-$ $7.52(\mathrm{~m}, 3 \mathrm{H}), 7.42-7.21(\mathrm{~m}, 4 \mathrm{H}), 6.95(\mathrm{~d}, J=8.0 \mathrm{~Hz}, 2 \mathrm{H}) ;{ }^{13} \mathrm{C}\left\{{ }^{1} \mathrm{H}\right\}$ NMR $\left(75 \mathrm{MHz}, \mathrm{DMSO}-d_{6}\right) \delta$ $178.2,136.8,136.2,134.8,134.1,133.5,133.2,131.8,129.2,128.5,128.2,128.0,127.9,127.3,127.2$, 127.1, 126.7, 124.2, 123.4, 121.2, 115.7, 113.0, 92.4, 88.3; HRMS (ESI/Q-TOF) m/z: [M + H] Calcd for $\mathrm{C}_{50} \mathrm{H}_{27} \mathrm{O}_{4} \mathrm{~S}_{2} 755.1351$; Found 755.1358.

\section{(S)-2,2'-((4-hydroxy-4-oxidodinaphtho[2,1-d:1',2'-f][1,3,2]dioxaphosphepine-2,6-diyl)bis(ethyne-}

2,1-diyl))bis(9H-thioxanthen-9-one) (1b). To a stirred suspension of (S)-22 (1 equiv, 0.04 mmol, 0.03 
$\mathrm{g})$ in distilled pyridine $(0.27 \mathrm{~mL})$ was added dropwise distilled $\mathrm{POCl}_{3}(2.0$ equiv, $0.08 \mathrm{mmol}, 7.5 \mu \mathrm{L})$ at room temperature. The solution mixture was heated at $60^{\circ} \mathrm{C}$ for several hours, monitoring the evolution by TLC. After cooling down to room temperature, water $(0.35 \mathrm{~mL})$ was added and the reaction mixture was stirred under reflux for $3 \mathrm{~h}$. Then aq. $\mathrm{HCl}(6 \mathrm{M}, 1.5 \mathrm{~mL})$ was added and the mixture was further heated at reflux with vigorous stirring for $1 \mathrm{~h}$. The dark reaction mixture was then extracted with $\mathrm{CH}_{2} \mathrm{Cl}_{2}(3 \times 20 \mathrm{~mL})$. The combined organic phases were washed with brine, dried over MgSO4, filtered and concentrated under reduced pressure. The residue was purified by flash column chromatography on silica gel using $\mathrm{CH}_{2} \mathrm{Cl}_{2} / \mathrm{MeOH}$ as eluent (gradient from 20:1 to 10:1). The obtained compound was taken up in $\mathrm{CH}_{2} \mathrm{Cl}_{2}(10 \mathrm{~mL})$ and vigorously washed with aq. $\mathrm{HCl}(4 \mathrm{M}, 3 \times 10 \mathrm{~mL})$. The organic layer was concentrated under reduced pressure to afford a crude residue, which was co-evaporated several times with toluene, and finally dried under high vacuum at $50{ }^{\circ} \mathrm{C}$ for several hours to afford (S)-1b as a yellow solid $(0.025 \mathrm{~g}, 0.03 \mathrm{mmol}, 78 \%) .[\alpha]_{\mathrm{D}}^{25}+168\left(c 1.0, \mathrm{CH}_{2} \mathrm{Cl}_{2}\right)$; IR (neat) $v\left(\mathrm{~cm}^{-1}\right): 1636,1590$, 1472, 1438, 1422, 1399, 1264, 1207, 1162, 1096, 1034, 962, 918, 892, 837; mp: 235-245 ${ }^{\circ} \mathrm{C} ;{ }^{1} \mathrm{H}\left\{{ }^{13} \mathrm{C}\right\}$ NMR (300 MHz, DMSO-d6) $\delta 8.62(\mathrm{~s}, 2 \mathrm{H}), 8.55(\mathrm{~s}, 2 \mathrm{H}), 8.48(\mathrm{~d}, J=7.9 \mathrm{~Hz}, 2 \mathrm{H}), 8.12(\mathrm{~d}, J=8.0 \mathrm{~Hz}$ 2H), $8.02-7.73(\mathrm{~m}, 8 \mathrm{H}), 7.58(\mathrm{dt}, J=15.0,7.4 \mathrm{~Hz}, 4 \mathrm{H}), 7.41(\mathrm{t}, J=7.5 \mathrm{~Hz}, 2 \mathrm{H}), 7.21(\mathrm{~d}, J=8.4 \mathrm{~Hz}$ $2 \mathrm{H}) ;{ }^{13} \mathrm{C}\left\{{ }^{1} \mathrm{H}\right\} \mathrm{NMR}\left(75 \mathrm{MHz}, \mathrm{CDCl}_{3}\right) \delta 179.0,146.3,146.2,137.0,135.0,134.6,132.6,132.2,132.0$ $131.7,131.0,129.8,128.6,128.3,128.2,127.3,127.0,126.12,126.0,125.8,121.8,121.2,116.2,93.8$ 85.7; ${ }^{31} \mathrm{P}\left\{{ }^{1} \mathrm{H}\right\}$ NMR (202 MHz, $\left.\mathrm{CDCl}_{3}\right) \delta$ 3.68; HRMS (ESI/Q-TOF) m/z: $[\mathrm{M}+\mathrm{H}]^{+}$Calcd for $\mathrm{C}_{50} \mathrm{H}_{26} \mathrm{O}_{6} \mathrm{~S}_{2} \mathrm{P}$ 817.0908; Found 817.0900.

\section{Preparation of Photocatalyst (1c):}

(R)-2-(2,2'-bis(methoxymethoxy)-[1,1'-binaphthalen]-3-yl)-9H-thioxanthen-9-one (13). To a stirred solution of $(\boldsymbol{R})-\mathbf{1 2}$ (1 equiv, $3.0 \mathrm{mmol}, 1.12 \mathrm{~g})$ in THF $(15 \mathrm{~mL})$ was added $n$-BuLi $(2.5 \mathrm{M}$ in hexanes, 1.1 equiv, $3.3 \mathrm{mmol}, 1.32 \mathrm{~mL})$ at $0{ }^{\circ} \mathrm{C}$. After stirring for $30 \mathrm{~min}$, freshly distilled $\mathrm{B}(\mathrm{OMe})_{3}(1.3$ equiv, 4 mmol, $440 \mu \mathrm{L})$ was added and the reaction mixture was stirred for $30 \mathrm{~min}$ at $0{ }^{\circ} \mathrm{C}$. Aq. $\mathrm{HCl}(4 \mathrm{M}, 5 \mathrm{~mL})$ was added and the reaction mixture was stirred for $30 \mathrm{~min}$ at room temperature. Water $(10 \mathrm{~mL})$ was 
added and the reaction mixture was extracted with EtOAc $(3 \times 15 \mathrm{~mL})$. The combined organic phases were washed with brine, dried over $\mathrm{MgSO}_{4}$, filtered and concentrated on a rotary evaporator. The residue was placed in a $250 \mathrm{~mL}$ round bottom flask and dried under high vacuum for $2 \mathrm{~h}$. Then a magnetic stir bar, 2-bromo-thioxanthone $\mathbf{8}^{14}$ (1.5 equiv, $4.5 \mathrm{mmol}, 1.3 \mathrm{~g}$ ), $\mathrm{K}_{2} \mathrm{CO}_{3}$ (6 equiv, $18 \mathrm{mmol}$, $2.5 \mathrm{~g})$ and $\mathrm{Pd}\left(\mathrm{PPh}_{3}\right)_{4}(0.1$ equiv, $0.3 \mathrm{mmol}, 0.35 \mathrm{~g})$ were added. The flask was purged with argon. A mixture of $\mathrm{THF} / \mathrm{H}_{2} \mathrm{O}(9: 1,84 \mathrm{~mL}$, freshly degassed by bubbling argon for $15 \mathrm{~min}$ in a ultrasonic bath) was added. The reaction mixture was heated for $12 \mathrm{~h}$ at $75^{\circ} \mathrm{C}$. After cooling down to room temperature, the reaction mixture was extracted by $\mathrm{CH}_{2} \mathrm{Cl}_{2}(3 \times 50 \mathrm{~mL})$. The combined organic phases were washed with brine, dried over $\mathrm{MgSO}_{4}$, filtered and concentrated under reduced pressure. The residue was purified by flash column chromatography on silica gel using PE/EtOAc as eluent (gradient from 15:1 to 5:1) to afford $(\boldsymbol{R})-\mathbf{1 3}$ as a yellow solid $(0.79 \mathrm{~g}, 1.35 \mathrm{mmol}, 45 \%$ over 2 steps $)$. $[\alpha]_{\mathrm{D}}^{25}+88$ (c 1.0 , $\mathrm{CH}_{2} \mathrm{Cl}_{2}$ ); IR (neat) $v\left(\mathrm{~cm}^{-1}\right): 1635,1591,1508,1471,1317,1261,1241,1148,1069,1032,1009,970$, 922, 795; mp: $120-140{ }^{\circ} \mathrm{C} ;{ }^{1} \mathrm{H}\left\{{ }^{13} \mathrm{C}\right\} \operatorname{NMR}\left(300 \mathrm{MHz}, \mathrm{CDCl}_{3}\right): \delta 8.90(\mathrm{~d}, J=1.9 \mathrm{~Hz}, 1 \mathrm{H}), 8.58(\mathrm{~d}, J=$ $7.8 \mathrm{~Hz}, 1 \mathrm{H}), 8.07-7.97(\mathrm{~m}, 2 \mathrm{H}), 7.93-7.75(\mathrm{~m}, 3 \mathrm{H}), 7.55(\mathrm{ddd}, J=7.7,7.3,2.6 \mathrm{~Hz}, 3 \mathrm{H}), 7.46-7.25$ (m, 3H), $7.25-7.16(\mathrm{~m}, 4 \mathrm{H}), 7.15-7.00(\mathrm{~m}, 1 \mathrm{H}), 5.07(\mathrm{dd}, J=23.9,6.9 \mathrm{~Hz}, 2 \mathrm{H}), 4.26(\mathrm{dd}, J=14.4$, $5.6 \mathrm{~Hz}, 2 \mathrm{H}), 3.18(\mathrm{~s}, 3 \mathrm{H}), 2.26(\mathrm{~s}, 3 \mathrm{H}),{ }^{13} \mathrm{C}\left\{{ }^{1} \mathrm{H}\right\} \mathrm{NMR}\left(75 \mathrm{MHz}, \mathrm{CDCl}_{3}\right): \delta 179.9,153.0,150.8,137.6$, $137.2,136.2,134.1,132.3,131.3,131.1,130.6,130.3,130.0,129.8,129.7,129.3,128.4,128.2,127.9$, $127.4,126.7,126.6,126.4,126.1,125.9,125.7,125.4,124.3,124.2,124.0,120.8,117.8,116.6,98.8$, 95.0, 56.2, 56.0; HRMS (ESI/Q-TOF) m/z: $[\mathrm{M}+\mathrm{H}]^{+}$Calcd for $\mathrm{C}_{37} \mathrm{H}_{29} \mathrm{O}_{5} \mathrm{~S}$ 585.1736; Found 585.1731.

(R)-2-(2,2'-dihydroxy-[1,1'-binaphthalen]-3-yl)-9H-thioxanthen-9-one (23). To a stirred solution of (R)-13 (1 equiv, $1.35 \mathrm{mmol}, 0.8 \mathrm{~g}$ ) in dioxane (45 mL) was slowly added $\mathrm{HCl}$ (12 $\mathrm{M}$ in $\mathrm{MeOH}, 24$ equiv, $2.7 \mathrm{~mL}$ ). After complete addition, the resulting suspension was stirred at $60{ }^{\circ} \mathrm{C}$ overnight. Upon completion (monitored by TLC), water $(25 \mathrm{~mL})$ was added at room temperature. The aqueous phase was extracted with $\mathrm{CH}_{2} \mathrm{Cl}_{2}(3 \times 25 \mathrm{~mL})$. The combined organic phases were washed with aq. $\mathrm{NaHCO}_{3}$ $(50 \mathrm{~mL})$, dried over $\mathrm{MgSO}_{4}$, filtered and concentrated under reduced pressure to afford $(\boldsymbol{R})-\mathbf{2 3}(0.64$ 
$\mathrm{g}, 1.3 \mathrm{mmol}, 95 \%)$ which was used in the next step without further purification. $[\alpha]_{\mathrm{D}}{ }^{25}+123(c 1.0$, $\left.\mathrm{CH}_{2} \mathrm{Cl}_{2}\right)$; IR (neat) $v\left(\mathrm{~cm}^{-1}\right): 1619,1588,1501,1436,1381,1326,1263,1175,1122,1078,1014,965$, 894; mp: $173-180{ }^{\circ} \mathrm{C} ;{ }^{1} \mathrm{H}\left\{{ }^{13} \mathrm{C}\right\} \mathrm{NMR}\left(500 \mathrm{MHz}, \mathrm{CD}_{3} \mathrm{CN}\right): \delta 9.09(\mathrm{~d}, J=1.9 \mathrm{~Hz}, 1 \mathrm{H}), 8.79(\mathrm{~d}, J=8.0$ $\mathrm{Hz}, 1 \mathrm{H}), 8.36(\mathrm{~s}, 1 \mathrm{H}), 8.32(\mathrm{dd}, J=8.4,2.1 \mathrm{~Hz}, 1 \mathrm{H}), 8.28-8.14(\mathrm{~m}, 3 \mathrm{H}), 8.07-7.92(\mathrm{~m}, 3 \mathrm{H}), 7.85-$ $7.75(\mathrm{~m}, 1 \mathrm{H}), 7.66-7.49(\mathrm{~m}, 6 \mathrm{H}), 7.39(\mathrm{~d}, J=8.3 \mathrm{~Hz}, 1 \mathrm{H}), 7.29-7.15(\mathrm{~m}, 2 \mathrm{H}), 6.80(\mathrm{~s}, 1 \mathrm{H}) ;{ }^{13} \mathrm{C}\left\{{ }^{1} \mathrm{H}\right\}$ $\operatorname{NMR}\left(75 \mathrm{MHz}, \mathrm{CDCl}_{3}\right): \delta 179.9,152.9,150.2,137.2,136.5,136.1,133.8,133.4,133.1,132.3,131.8$ $131.6,131.4,130.5,130.0,129.5,129.2,129.1,128.6,128.4,127.7,127.6,126.4,126.1,125.8,124.6$ 124.14 (2 peaks overlapping), 124.11 (2 peaks overlapping), 117.9, 112.0, 110.8; HRMS (ESI/Q-TOF) $\mathrm{m} / \mathrm{z}:[\mathrm{M}+\mathrm{H}]^{+}$Calcd for $\mathrm{C}_{33} \mathrm{H}_{21} \mathrm{O}_{3} \mathrm{~S}$ 497.1211; Found 497.1214.

\section{2-((4R)-4-hydroxy-4-oxidodinaphtho[2,1-d:1',2'-f][1,3,2]dioxaphosphepin-2-yl)-9H-thioxanthen-9-}

one (1c). To a stirred suspension of $(\boldsymbol{R})-23$ (1 equiv, $0.68 \mathrm{mmol}, 0.34 \mathrm{~g}$ ) in distilled pyridine $(4.5 \mathrm{~mL})$, was added dropwise distilled $\mathrm{POCl}_{3}(2.0$ equiv, $1.36 \mathrm{mmol}, 0.12 \mathrm{~mL})$. The resulting mixture was heated at $60{ }^{\circ} \mathrm{C}$ for several hours, monitoring the evolution by TLC. After $12 \mathrm{~h}$, the mixture was cooled down to room temperature, $\mathrm{H}_{2} \mathrm{O}(6 \mathrm{~mL})$ was added and the reaction mixture was strirred under reflux for $3 \mathrm{~h}$. Then, aq. $\mathrm{HCl}(6 \mathrm{M}, 22 \mathrm{~mL})$ was added and the reaction mixture was heated under reflux with vigorous stirring for $1 \mathrm{~h}$. The dark reaction mixture was then extracted with $\mathrm{CH}_{2} \mathrm{Cl}_{2}(3 \times 20 \mathrm{~mL})$. The combined organic phases were washed with brine, dried over $\mathrm{MgSO}_{4}$, filtered and concentrated under reduced pressure. The residue was purified by flash column chromatography on silica gel using $\mathrm{CH}_{2} \mathrm{Cl}_{2} / \mathrm{MeOH}$ as eluent (gradient from 20:1 to 10:1). The obtained compound was then taken up in $\mathrm{CH}_{2} \mathrm{Cl}_{2}(20 \mathrm{~mL})$ and vigorously washed with aq. $\mathrm{HCl}(4 \mathrm{M}, 3$ x $20 \mathrm{~mL})$. The organic layer was concentrated under reduced pressure to afford a crude residue, which was co-evaporated several times with toluene, and finally dried under high vacuum at $50{ }^{\circ} \mathrm{C}$ for several hours to afford $(\boldsymbol{R})-1 \mathrm{c}$ as a yellow solid $(0.3 \mathrm{~g}, 0.58$ mmol, 85\%). $[\alpha]_{\mathrm{D}}^{25}-62\left(c 1.0, \mathrm{CH}_{2} \mathrm{Cl}_{2}\right)$; IR (neat) $v\left(\mathrm{~cm}^{-1}\right): 1634,1590,1509,1460,1420,1392,1320$ $1284,1262,1216,1191,1068,1010,977,947,892 ; \mathrm{mp}: 272-284{ }^{\circ} \mathrm{C} ;{ }^{1} \mathrm{H}\left\{{ }^{13} \mathrm{C}\right\} \mathrm{NMR}(300 \mathrm{MHz}$ DMSO- $\left.d_{6}\right): \delta 8.79(\mathrm{t}, J=7.8 \mathrm{~Hz}, 1 \mathrm{H}), 8.53(\mathrm{~d}, J=7.8 \mathrm{~Hz}, 1 \mathrm{H}), 8.41-8.30(\mathrm{~m}, 2 \mathrm{H}), 8.20(\mathrm{dd}, J=8.3$, 
$5.3 \mathrm{~Hz}, 2 \mathrm{H}), 8.11(\mathrm{~d}, J=8.2 \mathrm{~Hz}, 1 \mathrm{H}), 8.00(\mathrm{~d}, J=8.5 \mathrm{~Hz}, 1 \mathrm{H}), 7.92(\mathrm{~d}, J=7.9 \mathrm{~Hz}, 1 \mathrm{H}), 7.82(\mathrm{t}, J=7.6$

$\mathrm{Hz}, 1 \mathrm{H}), 7.68-7.59(\mathrm{~m}, 1 \mathrm{H}), 7.54(\mathrm{dd}, J=8.7,5.0 \mathrm{~Hz}, 2 \mathrm{H}), 7.40(\mathrm{t}, J=7.7 \mathrm{~Hz}, 2 \mathrm{H}), 7.34-7.11(\mathrm{~m}$, $3 \mathrm{H}) .{ }^{13} \mathrm{C}\left\{{ }^{1} \mathrm{H}\right\} \mathrm{NMR}\left(75 \mathrm{MHz}, \mathrm{DMSO}-d_{6}\right): \delta 179.3,148.5,148.4,145.9,145.8,137.0,136.4,135.0$, 133.6, 133.0, 132.3, 132.1, 131.5, 131.4 (2 peaks overlapping), 131.3, 130.4, 129.7, 129.3, 129.1, 128.9, $128.7,127.5,127.4,127.3,127.2,126.9,126.6,126.5,126.3,125.8,122.8,121.9 ;{ }^{31} \mathrm{P}\left\{{ }^{1} \mathrm{H}\right\}$ NMR $(202$ $\mathrm{MHz}, \mathrm{CDCl}_{3}$ ): $\delta$ 3.71; HRMS (ESI/Q-TOF) $\mathrm{m} / \mathrm{z}:[\mathrm{M}+\mathrm{H}]^{+}$Calcd for $\mathrm{C}_{33} \mathrm{H}_{20} \mathrm{O}_{5} \mathrm{PS}$ 559.0769; Found 559.0778.

\section{General procedure for the enantioselective synthesis of azoles substituted vicinal diamines:}

Under argon, the $(\boldsymbol{E})$-enecarbamate $2\left(0.1 \mathrm{mmol}, 1.0\right.$ equiv) was dissolved in $\mathrm{CH}_{2} \mathrm{Cl}_{2}(0.4 \mathrm{~mL})$ in a flame-dried flask containing activated powdered $3 \AA$ molecular sieves $(20 \mathrm{mg})$. The solution was stirred at room temperature for $10 \mathrm{~min}$ before being cooled to $-45{ }^{\circ} \mathrm{C}$ and stirred for additional $10 \mathrm{~min}$. Dibenzyl azodicarboxylate (44.7 mg, $0.15 \mathrm{mmol}, 1.5$ equiv) dissolved in $\mathrm{CH}_{2} \mathrm{Cl}_{2}(0.3 \mathrm{~mL})$ was added and the reaction mixture was stirred for $10 \mathrm{~min}$. Then EtSH $(7 \mu \mathrm{L}, 0.1 \mathrm{mmol}, 1$ equiv) and finally the chiral catalyst 1 ( $0.01 \mathrm{mmol}, 0.1$ equiv) were added as a solution in $\mathrm{CH}_{2} \mathrm{Cl}_{2}(0.3 \mathrm{~mL})$ and the reaction mixture was stirred for $16 \mathrm{~h}$ at $-45^{\circ} \mathrm{C}$. Then, the suitable azole nucleophile was added $(0.3 \mathrm{mmol}, 3$ equiv) and the reaction was stirred at room temperature under an $\mathrm{O}_{2}$ atmosphere and blue light irradiation for $16 \mathrm{~h}$. Completion of the reaction was checked by TLC. The solvent was evaporated under reduced pressure and the residue was purified by flash column chromatography (PE/EtOAc 8:3).

Note: The presence of the Cbz groups create rotamers for the compounds 6. The NMR conduct at room temperature give spectra with broad signals. Running the NMR in deuterated acetonitrile $\left(\mathrm{CD}{ }_{3} \mathrm{CN}\right)$ instead of $\mathrm{CDCl}_{3}$ furnish acceptable spectra.

Dibenzyl 1-((1S,2R)-1-(((benzyloxy)carbonyl)amino)-1-(1H-pyrazol-1-yl)propan-2-yl)hydrazine1,2-dicarboxylate (6a). Following the general procedure, 6a was obtained from the corresponding $(E)$ - 
enecarbamate $\mathbf{2 a}^{8 \mathrm{~d}}(19.1 \mathrm{mg}, 0.1 \mathrm{mmol})$ and pyrazole $(20 \mathrm{mg}, 0.3 \mathrm{mmol})$. The crude product was purified by column chromatography on silica gel (PE/EtOAc 8:3) to afford 6a as a colourless oil (47 mg, 7:3 dr, 98/97\% ee) in $83 \%$ yield. $d r$ was determined by ${ }^{1} H$ NMR via integration of the singlets at 6.21 and 6.24 ppm; $[\alpha]_{\mathrm{D}}^{25}-6.5\left(\mathrm{c} 1.0, \mathrm{CH}_{2} \mathrm{Cl}_{2}\right)$; IR (neat) $v\left(\mathrm{~cm}^{-1}\right): 1699,1498,1402,1299,1215,1041$, 1016, 733, 695; ${ }^{1} \mathrm{H}\left\{{ }^{13} \mathrm{C}\right\} \mathrm{NMR}\left(500 \mathrm{MHz}, \mathrm{CD}_{3} \mathrm{CN}\right)$ mixture of diastereomers and rotamers: $\delta 7.91-$ $6.73(\mathrm{~m}, 19 \mathrm{H}), 6.24(\mathrm{~s}, 0.18 \mathrm{H}$, major diasteroisomer), $6.21(\mathrm{~s}, 0.45 \mathrm{H}$, minor diastereoisomer), 5.82 (s, $1 \mathrm{H}), 5.30-4.60(\mathrm{~m}, 7 \mathrm{H}), 1.30-1.12(\mathrm{~m}, 2 \mathrm{H}), 0.90-0.55(\mathrm{~m}, 1 \mathrm{H}) ;{ }^{13} \mathrm{C}\left\{{ }^{1} \mathrm{H}\right\} \mathrm{NMR}\left(126 \mathrm{MHz}, \mathrm{CDCl}_{3}\right)$ mixture of diastereoisomers and rotamers: $\delta$ 157.2, 156.7, 155.3, 149.7, 140,0, 139.1, 136.4, 136.1, $135.9,128.2,128.2,128.1,127.9,127.8,127.7,127.5,127.2,117.0,105.2,140.9,69.5,69.4,69.2,67.6$, 67.2, 67.0, 66.8, 66.7, 66.4, 66.2, 56.9, 55.8, 55.6, 13.7, 13.2, 12.6; HRMS (ESI/Q-TOF) m/z: $[\mathrm{M}+\mathrm{Na}]^{+}$

Calcd for $\mathrm{C}_{30} \mathrm{H}_{31} \mathrm{~N}_{5} \mathrm{O}_{6} \mathrm{Na} 580.2167$; Found 580.2168; enantiomeric excesses were determined by HPLC $\left(\right.$ Chiralpak IA, Heptane/i-PrOH $=85: 15$, flow rate $\left.=1.0 \mathrm{~mL} \cdot \mathrm{min}^{-1}, 214 \mathrm{~nm}\right):$ major diastereoisomer: $\mathrm{t}_{1}=$ $31.8 \mathrm{~min}$ (major enantiomer), $\mathrm{t}_{4}=86.9 \mathrm{~min}$ (minor enantiomer); minor diastereoisomer: $\mathrm{t}_{2}=39.2 \mathrm{~min}$ (major enantiomer), $\mathrm{t}_{3}=76.4 \mathrm{~min}$ (minor enantiomer).

\section{Dibenzyl 1-((1S,2R)-1-(((benzyloxy)carbonyl)amino)-1-(3,5-dimethyl-1H-pyrazol-1-yl)propan-2-}

yl)hydrazine-1,2-dicarboxylate (6b). Following the general procedure, $6 \mathbf{b}$ was obtained from the corresponding (E)-enecarbamate $2 \mathbf{a}(19.1 \mathrm{mg}, 0.1 \mathrm{mmol})$ and 3,5-dimethylpyrazole $(28.8 \mathrm{mg}, 0.3$ mmol). The crude product was purified by column chromatography on silica gel (PE/EtOAc 8:3) to afford $\mathbf{6 b}$ as a colorless oil (53 mg, 7:3 $d r, 98 / 98 \%$ ee) in 91\% yield. $d r$ was determined by ${ }^{1} H$ NMR via integration of the singlets at 5.76 and $5.69 \mathrm{ppm} ;[\alpha]_{\mathrm{D}}^{25}-9\left(\mathrm{c} 1.0, \mathrm{CH}_{2} \mathrm{Cl}_{2}\right)$; IR (neat) $v\left(\mathrm{~cm}^{-1}\right)$ : 1719 , 1498, 1455, 1407, 1306, 1027, 738, 696; ${ }^{1} \mathrm{H}\left\{{ }^{13} \mathrm{C}\right\} \quad \mathrm{NMR}\left(500 \mathrm{MHz}, \mathrm{CD}_{3} \mathrm{CN}\right)$ mixture of diastereoisomers and rotamers: $\delta 7.81(\mathrm{~s}, 1 \mathrm{H}), 7.34-7.23(\mathrm{~m}, 17 \mathrm{H}), 5.76(\mathrm{~s}, 0.7 \mathrm{H}$, major diastereoisomer), $5.69(\mathrm{~s}, 0.3 \mathrm{H}$, minor diastereoisomer), $5.12-5.01(\mathrm{~m}, 7 \mathrm{H}), 2.31-1.94(\mathrm{~m}, 6 \mathrm{H}), 2.31$ $-2.11(\mathrm{~m}, 6 \mathrm{H}), 1.28-0.88(\mathrm{~m}, 3 \mathrm{H}) ;{ }^{13} \mathrm{C}\left\{{ }^{1} \mathrm{H}\right\} \mathrm{NMR}\left(126 \mathrm{MHz}, \mathrm{CDCl}_{3}\right)$ mixture of diastereoisomers and 
rotamers: $\delta 156.6,156.1,155.6,149.7,149.3,136.0,135.6,128.7,128.6,128.5,128.4,128.3,128.2$, $128.1,127.9,127.7,105.6,68.8,68.6,68.2,67.9,68.0,67.5,67.3,65.3,65.0,13.7,13.5,10.9$; HRMS (ESI/Q-TOF) m/z: $[\mathrm{M}+\mathrm{H}]^{+}$Calcd for $\mathrm{C}_{32} \mathrm{H}_{36} \mathrm{~N}_{5} \mathrm{O}_{6}$ 586.2666; Found 586.2682; enantiomeric excesses were determined by HPLC (Chiralpak IA, Heptane $/ i$-PrOH = 95:5, flow rate $=1.0 \mathrm{~mL} \cdot \mathrm{min}^{-1}, 214 \mathrm{~nm}$ ): major diastereoisomer: $\mathrm{t}_{1}=44.0 \mathrm{~min}$ (minor enantiomer), $\mathrm{t}_{2}=47.0 \mathrm{~min}$ (major enantiomer); minor diastereoisomer: $\mathrm{t}_{3}=52.6 \mathrm{~min}$ (major enantiomer), $\mathrm{t}_{4}=70.5 \mathrm{~min}$ (minor enantiomer).

\section{Dibenzyl 1-((2R)-1-(((benzyloxy)carbonyl)amino)-1-(4-nitro-1H-pyrazol-1-yl)propan-2-}

yl)hydrazine-1,2-dicarboxylate (6c). Following the general procedure, 6c was obtained from the corresponding $(E)$-enecarbamate $2 \mathbf{a}(19.1 \mathrm{mg}, 0.1 \mathrm{mmol})$ and 4-nitro- $1 H$-pyrazole $(34.0 \mathrm{mg}, 0.3 \mathrm{mmol})$. The crude product was purified by column chromatography on silica gel (PE/EtOAc 8:3) to afford 6c as a colorless oil (57 mg, 1:1 dr, 99/99\% ee) in 95\% yield; $d r$ was determined by ${ }^{1} H$ NMR via integration of the multiplets at 1.3 and $1.0 \mathrm{ppm} ;[\alpha]_{\mathrm{D}}^{25}-5\left(\mathrm{c} 1.0, \mathrm{CH}_{2} \mathrm{Cl}_{2}\right)$; IR (neat) $v\left(\mathrm{~cm}^{-1}\right)$ : 1704 , $1510,1455,1406,1304,1258,1226,1024,872,817,797,752 ;{ }^{1} \mathrm{H}\left\{{ }^{13} \mathrm{C}\right\} \mathrm{NMR}\left(500 \mathrm{MHz}, \mathrm{CD}{ }_{3} \mathrm{CN}\right)$ mixture of diastereoisomers and rotamers: $\delta 8.58(\mathrm{~m}, 1 \mathrm{H}), 8.27-8.13(\mathrm{~m}, 1 \mathrm{H}), 8.01-7.79(\mathrm{~m}, 1 \mathrm{H}), 7.48-$ $7.05(\mathrm{~m}, 16 \mathrm{H}), 6.05-5.75(\mathrm{~m}, 7 \mathrm{H}), 5.23-4.60(\mathrm{~m}, 7 \mathrm{H}), 1.32-1.31(\mathrm{~m}, 1.5 \mathrm{H}$ diastereoisomer 1$), 1.03$ $-0.91(\mathrm{~m}, 1.5 \mathrm{H}$, diastereoisomer 2$) ;{ }^{13} \mathrm{C}\left\{{ }^{1} \mathrm{H}\right\} \mathrm{NMR}\left(75 \mathrm{MHz}, \mathrm{CDCl}_{3}\right)$ mixture of diastereoisomers and rotamers: $\delta 156.9,156.6,156.0,136.8,135.8,135.2,131.9,129.2,128.7,128,6,128.4,128.3,128.1$, 128.0 71.3, 68.7, 68.4, 68.2, 68.0, 67.7, 56.2, 14.3; HRMS (ESI/Q-TOF) m/z: [M + Na $]^{+}$Calcd for $\mathrm{C}_{30} \mathrm{H}_{30} \mathrm{~N}_{6} \mathrm{O}_{8} \mathrm{Na}$ 625.1997; Found 625.1986; enantiomeric excesses weredetermined by HPLC (Chiralpak IA, Heptane $/ i-\mathrm{PrOH}=85: 15$, flow rate $\left.=1.0 \mathrm{~mL} \cdot \mathrm{min}^{-1}, 214 \mathrm{~nm}\right)$ :diastereoisomer $1: \mathrm{t}_{1}=39.0$ min $($ major enantiomer), $t_{2}=45.4 \min$ (minor enantiomer); minor diastereoisomer $2: t_{3}=55.0$ min (major enantiomer), $\mathrm{t}_{4}=70.8 \mathrm{~min}$ (minor enantiomer).

\section{Dibenzyl 1-((2R)-1-(((benzyloxy)carbonyl)amino)-1-(3-(trifluoromethyl)-1H-pyrazol-1-yl)propan-} 2-yl)hydrazine-1,2-dicarboxylate (6d). Following the general procedure, $6 \mathrm{~d}$ was obtained from the 
corresponding $(E)$-enecarbamate $2 \mathrm{a}(19.1 \mathrm{mg}, 0.1 \mathrm{mmol})$ and 3-(trifluoromethyl)pyrazole $(40.8 \mathrm{mg}$, $0.3 \mathrm{mmol}$ ). The crude product was purified by column chromatography on silica gel (PE/EtOAc 8:3) to afford $6 \mathrm{~d}$ as a colorless oil (54 mg, 1:1 $d r, 99 / 99 \%$ ee) in $87 \%$ yield; $d r$ was determined by ${ }^{19} F N M R$ via integration of the singlets at -61.8 and $-62.0 \mathrm{ppm} ;[\alpha]_{\mathrm{D}} \mathrm{D}^{25}-27\left(\mathrm{c} 1.0, \mathrm{CH}_{2} \mathrm{Cl}_{2}\right)$; IR (neat) $\vee\left(\mathrm{cm}^{-1}\right)$ : $1702,1525,1455,1409,1298,1258,1218,1131,1026,967,794,739 ;{ }^{1} \mathrm{H}\left\{{ }^{13} \mathrm{C}\right\} \mathrm{NMR}\left(500 \mathrm{MHz}, \mathrm{CDCl}_{3}\right)$ mixture of diastereoisomers and rotamers: $\delta 7.64-7.49(\mathrm{~m}, 1 \mathrm{H}), 7.33-7.26(\mathrm{~m}, 14 \mathrm{H}), 7.13(\mathrm{~s}, 1 \mathrm{H})$, $7.00-6.53(\mathrm{~m}, 1 \mathrm{H}), 6.84-6.40(\mathrm{~m}, 1 \mathrm{H}), 6.00-5.60(\mathrm{~m}, 1 \mathrm{H}), 5.29-4.80(\mathrm{~m}, 7 \mathrm{H}), 1.32-1.37(\mathrm{~m}$, 1.4H), $1.27-1.02(\mathrm{~m}, 1.6 \mathrm{H}) ;{ }^{13} \mathrm{C}\left\{{ }^{1} \mathrm{H}\right\} \mathrm{NMR}\left(126 \mathrm{MHz}, \mathrm{CDCl}_{3}\right)$ mixture of diastereoisomers and rotamers: $\delta 156.4,155.9,155.5,135.6,135.4,134.8,131.7,128.8,128.7,128.6,128.4,128.3,127.7$, 122.2, 104.5, 70.8, 70.4, 69.7, 68.6, 68.3, 68.0, 67.8, 67.1, 56.6, 14.2, 13.7; ${ }^{19} \mathrm{~F}\left\{{ }^{1} \mathrm{H}\right\}$ NMR $(282 \mathrm{MHz}$, $\left.\mathrm{CDCl}_{3}\right) \delta-61.8(\mathrm{~s}, 1.5 \mathrm{~F}$, diastereoisomer 1), -62.0 (s, 1.5F, diastereoisomer 2); HRMS (ESI/Q-TOF) $\mathrm{m} / \mathrm{z}:[\mathrm{M}+\mathrm{H}]^{+}$Calcd for $\mathrm{C}_{31} \mathrm{H}_{31} \mathrm{~F}_{3} \mathrm{~N}_{5} \mathrm{O}_{6}$ 626.2189; Found 626.2221; enantiomeric excesses were determined by HPLC (Chiralpak IA, Heptane $/ \mathrm{i}-\mathrm{PrOH}=85: 15$, flow rate $=1.0 \mathrm{~mL} \cdot \mathrm{min}^{-1}, 214 \mathrm{~nm}$ ): minor diastereoisomer: $\mathrm{t}_{1}=13.01 \mathrm{~min}$ (major enantiomer), $\mathrm{t}_{3}=20.13 \mathrm{~min}$ (minor enantiomer); major diastereoisomer: $\mathrm{t}_{2}=14.7 \mathrm{~min}$ (minor enantiomer), $\mathrm{t}_{4}=34.7 \mathrm{~min}$ (major enantiomer).

\section{Dibenzyl 1-((1S,2R)-1-(((benzyloxy)carbonyl)amino)-1-(1H-indazol-1-yl)propan-2-yl)hydrazine-}

1,2-dicarboxylate (6e). Following the general procedure, $6 \mathrm{e}$ was obtained from the corresponding Eenecarbamate $2 \mathrm{a}(19.1 \mathrm{mg}, 0.1 \mathrm{mmol})$ and indazole $(35.4 \mathrm{mg}, 0.3 \mathrm{mmol})$. The crude product was purified by column chromatography on silica gel (PE/EtOAc 8:3) to afford $6 \mathbf{e}$ as a colorless oil (52 mg, 3:1 $d r, 99 / 92 \%$ ee) in $85 \%$ yield; $d r$ was determined by ${ }^{1} H N M R$ via integration of the singlets at 8.02 and 7.99 ppm; $[\alpha] \mathrm{D}^{25}-15$ (c 1.0, $\left.\mathrm{CH}_{2} \mathrm{Cl}_{2}\right)$; IR (neat) $\vee(\mathrm{cm}-1)$ : 1701, 1618, 1498, 1455, 1406, 1294, 1216, 1155, 1118, 1026; ${ }^{1} \mathrm{H}\left\{{ }^{13} \mathrm{C}\right\}$ NMR $\left(500 \mathrm{MHz}, \mathrm{CD}_{3} \mathrm{CN}\right)$ mixture of diastereoisomers and 
rotamers: $\delta 8.02(\mathrm{~s}, 0.7 \mathrm{H}$, major diastereoisomer), 7.99 (s, 0.3H, minor diastereoisomer), $7.79-7.52$ (m, 2H ), $7.36-7.28(\mathrm{~m}, 16 \mathrm{H}), 7.18-7.14(\mathrm{~m}, 1 \mathrm{H}), 7.09(\mathrm{~m}, 1 \mathrm{H}), 6.93(\mathrm{~m}, 1 \mathrm{H}), 6.30-6.12(\mathrm{~m}, 1 \mathrm{H})$, $5.30-4.75(\mathrm{~m}, 7 \mathrm{H}), 1.37-1.27(\mathrm{~m}, 2 \mathrm{H}), 0.91-0.86(\mathrm{~m}, 1 \mathrm{H}) ;{ }^{13} \mathrm{C}\left\{{ }^{1} \mathrm{H}\right\} \mathrm{NMR}\left(75 \mathrm{MHz}, \mathrm{CDCl}_{3}\right)$ mixture of diastereoisomers and rotamers: $\delta 156.6,156.3,155.8,135.4,135.0,128.5,128.4,128.2,128.1$, 127.8, 127.6, 127.4, 126.9, 126.8, 123.8, 122.3, 121.4, 121.2, 121.0, 120.6, 117.7, 109.9, 109.1 , 68.6, 67.9, 67.3, 67.0, 56.4, 14.1; HRMS (ESI/Q-TOF) m/z: $[\mathrm{M}+\mathrm{H}]^{+}$Calcd for $\mathrm{C}_{34} \mathrm{H}_{34} \mathrm{~N}_{5} \mathrm{O}_{6} 608.2509$; Found 608.2543; enantiomeric excesses were determined by HPLC (Chiralpak IA, Heptane/ $i$-PrOH = 80:20, flow rate $=1.0 \mathrm{~mL} \cdot \mathrm{min}^{-1}, 214 \mathrm{~nm}$ ): major diastereoisomer: $\mathrm{t}_{1}=37.6 \mathrm{~min}$ (minor enantiomer), $\mathrm{t}_{3}=80.9$ min (major enantiomer); minor diastereoisomer: $\mathrm{t}_{2}=61.0 \mathrm{~min}$ (major enantiomer), $\mathrm{t}_{4}=98.7$ $\min$ (minor enantiomer).

\section{Dibenzyl 1-((1S,2R)-1-(((benzyloxy)carbonyl)amino)-1-(1H-pyrazol-1-yl)pentan-2-yl)hydrazine-} 1,2-dicarboxylate (6f). Following the general procedure, $6 \mathbf{f}$ was obtained from the corresponding $(E)$ enecarbamate $2 \mathbf{b}(21.9 \mathrm{mg}, 0.1 \mathrm{mmol})$ and pyrazole $(20 \mathrm{mg}, 0.3 \mathrm{mmol})$. The crude product was purified by column chromatography on silica gel (PE/EtOAc 8:3) to afford $\mathbf{6} \mathbf{f}$ as a colorless oil (44 $\mathrm{mg}, 7: 3 d r, 99 / 99 \%$ ee) in $76 \%$ yield; $d r$ was determined by ${ }^{l} H$ NMR via integration of the singlets at 6.22 and $6.10 \mathrm{ppm} ;[\alpha]_{\mathrm{D}}^{25}-5\left(c \mathrm{c} .0, \mathrm{CH}_{2} \mathrm{Cl}_{2}\right) ; \mathrm{IR}$ (neat) $v(\mathrm{~cm}-1): 1704,1498,1455,1400,1258,1218$, 1145, 1040, 1027, 917, 801; ${ }^{1} \mathrm{H}\left\{{ }^{13} \mathrm{C}\right\}$ NMR $\left(300 \mathrm{MHz}, \mathrm{CDCl}_{3}\right)$ mixture of diastereoisomers and rotamers: $\delta 7.76-6.93(\mathrm{~m}, 18 \mathrm{H}), 6.78-6.37(\mathrm{~m}, 1 \mathrm{H}), 6.22(\mathrm{~s}, 0.4 \mathrm{H}$, minor diastereoisomer), $6.10(\mathrm{~s}$, 0.6H, major diastereoisomer), $6.02-5.71(\mathrm{~m}, 1 \mathrm{H}), 5.35-4.48(\mathrm{~m}, 7 \mathrm{H}), 1.81-1.03(\mathrm{~m}, 4 \mathrm{H}), 0.97-$ $0.64(\mathrm{~m}, 3 \mathrm{H}) ;{ }^{13} \mathrm{C}\left\{{ }^{1} \mathrm{H}\right\}$ NMR $\left(126 \mathrm{MHz}, \mathrm{CDCl}_{3}\right)$ mixture of diastereoisomers and rotamers: $\delta 156.7$, $156.1,155.5,140.9,140.2,139.7,135.8,135.7,135.6,135.5,130.2,130.1,128.5,128.4,128.3,128.2$, $127.7,105.8,105.6,69.6,69.1,68.6,68.2,67.9,67.6,61.0,30.4,29.6,24.4,19.6,19.4,19.2,14.0,13.7$; HRMS (ESI/Q-TOF) m/z: $[\mathrm{M}+\mathrm{H}]^{+}$Calcd for $\mathrm{C}_{32} \mathrm{H}_{36} \mathrm{~N}_{5} \mathrm{O}_{6}$ 586.2666; Found 586.2669; enantiomeric 
excess were determined by HPLC (Chiralpak IB, Heptane/ $i$-PrOH = 98:2, flow rate = 1.0 mL. $\mathrm{min}^{-1}, 214$ $\mathrm{nm}$ ): minor diastereoisomer: $\mathrm{t}_{1}=31.1 \mathrm{~min}$ (minor enantiomer), $\mathrm{t}_{4}=59.1 \mathrm{~min}$ (major enantiomer); major diastereoisomer: $\mathrm{t}_{2}=34.7 \mathrm{~min}$ (minor enantiomer), $\mathrm{t}_{3}=44.0 \mathrm{~min}$ (major enantiomer).

\section{Dibenzyl 1-((2R)-1-(((benzyloxy)carbonyl)amino)-1-(3,5-dimethyl-1H-pyrazol-1-yl)pentan-2-}

yl)hydrazine-1,2-dicarboxylate (6g). Following the general procedure, $6 \mathrm{~g}$ was obtained from the corresponding $(E)$-enecarbamate $2 \mathrm{~b}(21.9 \mathrm{mg}, 0.1 \mathrm{mmol})$ and 3,5-dimethylpyrazole $(28.8 \mathrm{mg}, 0.3$ mmol). The crude product was purified by column chromatography on silica gel (PE/EtOAc 8:3) to afford $6 \mathrm{~g}$ as a colorless oil (49 $\mathrm{mg}, 1: 1 d r$,99/99\% ee) in 80\% yield; $d r$ was determined by ${ }^{1} H N M R$ via integration of the multiplets at 0.89 and $0.79 \mathrm{ppm} ;[\alpha]_{\mathrm{D}}^{25}-25\left(c 1.0, \mathrm{CH}_{2} \mathrm{Cl}_{2}\right)$; IR (neat) $v(\mathrm{~cm}-1)$ : 1705, 1498, 1455, 1404, 1289, 1214, 1162, 1040, 1027, 911, 801; ${ }^{1} \mathrm{H}\left\{{ }^{13} \mathrm{C}\right\} \mathrm{NMR}\left(500 \mathrm{MHz}, \mathrm{CD}{ }_{3} \mathrm{CN}\right)$ mixture of diastereoisomers and rotamers: $\delta 7.51-7.07(\mathrm{~m}, 16 \mathrm{H}), 6.76-6.49(\mathrm{~m}, 0.5 \mathrm{H}), 5.82-5.58$ $(\mathrm{m}, 1.5 \mathrm{H}), 5.26-5.04(\mathrm{~m}, 6 \mathrm{H}), 4.75-4.50(\mathrm{~m}, 1 \mathrm{H}), 2.42-2.10(\mathrm{~m}, 7 \mathrm{H}), 1.54-1.10(\mathrm{~m}, 4 \mathrm{H}), 0.90-$ $0.87\left(\mathrm{~m}, 1.5 \mathrm{H}\right.$, diastereoisomer 1), $0.87-0.76(\mathrm{~m}, 1.5 \mathrm{H}$, diastereoisomer 2$) ;{ }^{13} \mathrm{C}\left\{{ }^{1} \mathrm{H}\right\} \mathrm{NMR}(126 \mathrm{MHz}$, $\left.\mathrm{CDCl}_{3}\right)$ mixture of diastereoisomers and rotamers: $\delta 156.6,156.2,155.5,149.4,135.9,128.6,128.5$, $128.4,128.2,128.1,127.7,127.08,106.0,105.9,105.5,69.7,69.3,68.7,67.9,67.4,65.4,64.9,64.5$, 61.6, 60.5, 30.8, 30.4, 29.5, 19.7, 19.5, 19.3, 19.2, 14.0, 13.9, 13.7, 13.5, 10.9;; HRMS (ESI/Q-TOF) m/z: $[\mathrm{M}+\mathrm{H}]^{+}$Calcd for $\mathrm{C}_{34} \mathrm{H}_{40} \mathrm{~N}_{5} \mathrm{O}_{6}$ 614.2979; Found 614.2981; enantiomeric excesses were determined by HPLC (Chiralpak IA, Heptane $/ i-\mathrm{PrOH}=95: 5$, flow rate $=1.0 \mathrm{~mL} \cdot \mathrm{min}^{-1}, 214 \mathrm{~nm}$ ): major diastereoisomer: $\mathrm{t}_{1}=18.7 \mathrm{~min}$ (major enantiomer), $\mathrm{t}_{4}=48.5 \mathrm{~min}$ (minor enantiomer); minor diastereoisomer: $\mathrm{t}_{2}=26.7 \mathrm{~min}$ (major enantiomer), $\mathrm{t}_{3}=35.1 \mathrm{~min}$ (minor enantiomer).

Dibenzyl 1-((1S,2R)-1-(((benzyloxy)carbonyl)amino)-1-(4-bromo-1H-pyrazol-1-yl)pentan-2yl)hydrazine-1,2-dicarboxylate (6h). Following the general procedure, $6 \mathrm{~h}$ was obtained from the 
corresponding $(E)$-enecarbamate $\mathbf{2 b}(21.9 \mathrm{mg}, 0.1 \mathrm{mmol})$ and 4-bromopyrazole (44.1 $\mathrm{mg}, 0.3 \mathrm{mmol})$. The crude product was purified by column chromatography on silica gel (PE/EtOAc 8:3) to afford $\mathbf{6 h}$ as a colorless oil (49 mg, 3:2 $d r, 97 / 98 \%$ ee) in 74\% yield; $d r$ was determined by ${ }^{1} H$ NMR via integration of the multiplets at 0.86 and $0.76 \mathrm{ppm} ;[\alpha] \mathrm{D}^{25}-4\left(c 1.0, \mathrm{CH}_{2} \mathrm{Cl}_{2}\right)$; IR 1697, 1498, 1455, 1407, 1258, 1215, 1027, 952, 910, 798; ${ }^{1} \mathrm{H}\left\{{ }^{13} \mathrm{C}\right\}$ NMR (500 MHz, $\left.\mathrm{CD}_{3} \mathrm{CN}\right)$ mixture of diastereoisomers and rotamers: $\delta 7.82-6.62(\mathrm{~m}, 19 \mathrm{H}), 6.00-5.20(\mathrm{~m}, 1 \mathrm{H}), 5.29-4.86(\mathrm{~m}, 6 \mathrm{H}), 4.65-4.47(\mathrm{~m}, 1 \mathrm{H})$, $1.60-1.05(\mathrm{~m}, 4 \mathrm{H}), 0.88-0.84(\mathrm{~m}, 1.2 \mathrm{H}$, minor diastereoisomer), $0.84-0.74(\mathrm{~m}, 1.8 \mathrm{H}$, major diastereoisomer); ${ }^{13} \mathrm{C}\left\{{ }^{1} \mathrm{H}\right\} \mathrm{NMR}\left(126 \mathrm{MHz}, \mathrm{CDCl}_{3}\right)$ mixture of diastereoisomers and rotamers: $\delta$ 156.6, 156.3, 156.0, 141.5, 140.8, 140.6, 135.7, 135.7, 135.5, 130.3, 129.8, 128.8, 128.73, 128.66, $128.58,128.51,128.4,128.0,127.8,110.1,93.6,69.4,69.0,68.5,68.2,67.8,67.5,60.7,30.3,29.8$, 19.6, 19.4, 13.7; HRMS (ESI/Q-TOF) m/z: $[\mathrm{M}+\mathrm{Na}]^{+}$Calcd for $\mathrm{C}_{32} \mathrm{H}_{34} \mathrm{BrN}_{5} \mathrm{O}_{6} \mathrm{Na}$ 686.1590; Found 686.1606; enantiomeric excesses were determined by HPLC (Chiralpak IC, Heptane/ $i$-PrOH = 95:5, flow rate $\left.=1.0 \mathrm{~mL} \cdot \mathrm{min}^{-1}, 214 \mathrm{~nm}\right)$ : minor diastereoisomer: $\mathrm{t}_{1}=12.2 \mathrm{~min}$ (minor enantiomer), $\mathrm{t}_{2}=$ $14.7 \mathrm{~min}$ (major enantiomer); major diastereoisomer: $\mathrm{t}_{3}=18.8 \mathrm{~min}$ (major enantiomer), $\mathrm{t}_{4}=28.6 \mathrm{~min}$ (minor enantiomer).

Dibenzyl 1-((1S,2R)-1-(((benzyloxy)carbonyl)amino)-1-(3-(trifluoromethyl)-1H-pyrazol-1-yl)pentan2-yl)hydrazine-1,2-dicarboxylate (6i). Following the general procedure, $6 \mathbf{i}$ was obtained from the corresponding (E)-enecarbamate $2 \mathrm{~b}(21.9 \mathrm{mg}, 0.1 \mathrm{mmol})$ and 3-(trifluoromethyl)pyrazole (40.8 mg, $0.3 \mathrm{mmol}$ ). The crude product was purified by column chromatography on silica gel (PE/EtOAc 8:3) to afford $6 \mathbf{i}$ a colorless oil (51 mg, 7:3 $d r, 96 / 96 \%$ ee) in 79\% yield; $d r$ was determined by ${ }^{19} F N M R$ via integration of the singlets at -61.8 and $-61.9 \mathrm{ppm} ;[\alpha]_{\mathrm{D}}{ }^{25}-5\left(\mathrm{c} 1.0, \mathrm{CH}_{2} \mathrm{Cl}_{2}\right)$; IR (neat) $v(\mathrm{~cm}-1)$ : 1714, 1498, 1455, 1407, 1294, 1255, 1169, 1135, 1028, 966, 741, 696; ${ }^{1} \mathrm{H}\left\{{ }^{13} \mathrm{C}\right\}$ NMR $(500 \mathrm{MHz}$, 
$\left.\mathrm{CDCl}_{3}\right)$ mixture of diastereoisomers and rotamers: $\delta 7.75-7.51(\mathrm{~m}, 1 \mathrm{H}), 7.48-7.05(\mathrm{~m}, 15 \mathrm{H}), 6.80-$ $6.59(\mathrm{~m}, 1 \mathrm{H}), 6.56-6.38(\mathrm{~m}, 1 \mathrm{H}), 5.90-5.75(\mathrm{~m}, 1 \mathrm{H}), 5.34-4.71(\mathrm{~m}, 6 \mathrm{H}), 4.70-4.51(\mathrm{~m}, 1 \mathrm{H}), 1.61$ $-1.20(\mathrm{~m}, 4 \mathrm{H}), 0.90-0.50(\mathrm{~m}, 3 \mathrm{H}) ;{ }^{13} \mathrm{C}\left\{{ }^{1} \mathrm{H}\right\} \mathrm{NMR}\left(126 \mathrm{MHz}, \mathrm{CDCl}_{3}\right)$ mixture of diastereoisomers and rotamers $\delta: 156.4,156.1,155.5,136.3,135.6,135.5,132.4,131.7,128.7,128.5,128.4,128.4,128.3$, $128.2,128.0,104.5,104.2,69.2,69.0,68.6,68.4,67.9,67.1,30.2,29.8,19.6,19.3,13.9,13.7 .{ }^{19} \mathrm{~F}\left\{{ }^{1} \mathrm{H}\right\}$ NMR (282 MHz, CDCl $)$ $\delta$-61.8 (s, 2H, major diastereosiomer), -61.9 (s, 1H, minor diastereoisomer); HRMS (ESI/Q-TOF) m/z: $[\mathrm{M}+\mathrm{Na}]^{+}$Calcd for $\mathrm{C}_{33} \mathrm{H}_{34} \mathrm{~F}_{3} \mathrm{~N}_{5} \mathrm{O}_{6} \mathrm{Na}$ 676.2325; Found 676.2353; enantiomeric excesses were determined by HPLC (Chiralpak IA, Heptane/ $i$-PrOH = 90:10, flow rate $\left.=1.0 \mathrm{~mL} \cdot \mathrm{min}^{-1}, 214 \mathrm{~nm}\right):$ minor diastereoisomer: $\mathrm{t}_{1}=16.2 \mathrm{~min}$ (minor enantiomer), $\mathrm{t}_{2}=18.3 \mathrm{~min}$ (major enantiomer); major diastereoisomer: $\mathrm{t}_{3}=31.9 \mathrm{~min}$ (major enantiomer), $\mathrm{t}_{4}=35.8 \mathrm{~min}$ (minor enantiomer).

Dibenzyl 1-((1S,2R)-1-(((benzyloxy)carbonyl)amino)-1-(1H-indazol-1-yl)pentan-2-yl)hydrazine-1,2dicarboxylate $(\mathbf{6 j})$. Following the general procedure, $\mathbf{6 j}$ was obtained from the corresponding $(E)$ enecarbamate $2 \mathrm{~d}(21.9 \mathrm{mg}, 0.1 \mathrm{mmol})$ and indazole $(35.4 \mathrm{mg}, 0.3 \mathrm{mmol})$. The crude product was purified by column chromatography on silica gel (PE/EtOAc 8:3) to afford $\mathbf{6 j}$ as a colorless oil (32 mg, 7:3 $d r, 99 / 99 \%$ ee) in 50\% yield; $d r$ was determined by ${ }^{1} H$ NMR via integration of the multiplets at 0.86 and 0.69 ppm; $[\alpha] \mathrm{D}^{25}-6\left(c 1.0, \mathrm{CH}_{2} \mathrm{Cl}_{2}\right)$; IR (neat) $\vee(\mathrm{cm}-1): 1703,1629,1515,1498,1455$, 1404, 1213, 1115, 1027, 1042, 909, 848, 734; ${ }^{1} \mathrm{H}\left\{{ }^{13} \mathrm{C}\right\}$ NMR $\left(500 \mathrm{MHz}, \mathrm{CD}_{3} \mathrm{CN}\right)$ mixture of diastereoisomers and rotamers: $\delta 8.63-6.80(\mathrm{~m}, 22 \mathrm{H}), 6.50-6.00(\mathrm{~m}, 1 \mathrm{H}), 5.44-4.16(\mathrm{~m}, 7 \mathrm{H}), 1.64$ - $1.00(\mathrm{~m}, 4 \mathrm{H})$, ) $, 0.90-0.84(\mathrm{~m}, \mathrm{H}$, major diastereoisomer $), 0.84-0.66(\mathrm{~m}, 1 \mathrm{H}$, minor diastereoisomer); ${ }^{13} \mathrm{C}\left\{{ }^{1} \mathrm{H}\right\} \mathrm{NMR}\left(75 \mathrm{MHz}, \mathrm{CD}_{3} \mathrm{CN}\right)$ mixture of diastereoisomers and rotamers: $\delta$ 156.7, 156.6, 149.9, 137.1, 135.1, 129.4, 129.3, 129.1, 129.0, 128.9, 128.8, 128.6, 128.2, 127.6, 
$127.3,124.8,122.7,122.0,121.7,121.5,118.3,110.9,71.5,69.9,68.6,68.3,68.1,67.8,67.5,62.6$,

61.5, 31.0, 20.1, 19.9, 14.1, 13.7, 13.3; HRMS (ESI/Q-TOF) m/z: $[\mathrm{M}+\mathrm{Na}]^{+}$Calcd for $\mathrm{C}_{36} \mathrm{H}_{37} \mathrm{~N}_{5} \mathrm{O}_{6} \mathrm{Na}$

658.2621; Found 658.2636; enantiomeric excesses were determined by HPLC (Chiralpak IC, Heptane $/ i-\mathrm{PrOH}=95: 5$, flow rate $\left.=1.0 \mathrm{~mL} \cdot \mathrm{min}^{-1}, 300 \mathrm{~nm}\right):$ minor diastereoisomer: $\mathrm{t}_{1}=39.1 \mathrm{~min}$ (major enantiomer), $\mathrm{t}_{2}=65.1 \mathrm{~min}$ (minor enantiomer); major diastereoisomer: $\mathrm{t}_{3}=75.8 \mathrm{~min}$ (major enantiomer), $\mathrm{t}_{4}=90.5 \mathrm{~min}$ (minor enantiomer).

Dibenzyl 1-((1S,2R)-1-(((benzyloxy)carbonyl)amino)-3-methyl-1-(1H-pyrazol-1-yl)butan-2yl)hydrazine-1,2-dicarboxylate (6k). Following the general procedure, $6 \mathbf{k}$ was obtained from the corresponding (E)-enecarbamate $2 \mathrm{c}(21.9 \mathrm{mg}, 0.1 \mathrm{mmol})$ and pyrazole $(20 \mathrm{mg}, 0.3 \mathrm{mmol})$. The crude product was purified by column chromatography on silica gel (PE/EtOAc 8:3) to afford $\mathbf{6 k}$ as a colorless oil (20 mg, 7:3 $d r, 98 / 92 \%$ ee) in $30 \%$ yield; $d r$ was determined by ${ }^{1} H$ NMR via integration of the multiplets at 4.57 and $4.34 \mathrm{ppm} ;[\alpha]_{\mathrm{D}}^{25}-19\left(\mathrm{c} 1.0, \mathrm{CH}_{2} \mathrm{Cl}_{2}\right)$; IR (neat) $\vee(\mathrm{cm}-1): 1715,1498$, 1455, 1400, 1299, 1260, 1216, 1045, 738; ${ }^{1} \mathrm{H}\left\{{ }^{13} \mathrm{C}\right\} \quad \mathrm{NMR}\left(500 \mathrm{MHz}, \mathrm{CDCl}_{3}\right)$ mixture of diastereoisomers and rotamers: $\delta 7.64-7.03(\mathrm{~m}, 16 \mathrm{H}), 6.80-6.50(\mathrm{~m}, 1 \mathrm{H}), 6.23-6.18(\mathrm{~m}, 1 \mathrm{H}) 6.13-$ $6.07(\mathrm{~m}, 1 \mathrm{H}), 5.32-4.77(\mathrm{~m}, 8 \mathrm{H}), 4.71-4.50(\mathrm{~m}, 0.65 \mathrm{H}$, major diastereoisomer), $4.50-4.10(\mathrm{~m}$, 0.35H, minor diastereoisomer), $2.14-1.80(\mathrm{~m}, 1 \mathrm{H}), 1.21-0.88(\mathrm{~m}, 6 \mathrm{H}) .{ }^{13} \mathrm{C}\left\{{ }^{1} \mathrm{H}\right\} \mathrm{NMR}(126 \mathrm{MHz}$, $\mathrm{CDCl}_{3}$ ) mixture of diastereoisomers and rotamers: $\delta 157.0,156.5,156.0,140.6,139.7,135.9,135.7$, $135.4,130.1,129.8,128.74,128.68,128.59,128.4,128.3,128.2,127.8,105.6,69.7,68.7,68.4,68.2$, 68.1, 67.6, 67.1, 28.8, 28.2, 19.8, 19.2 HRMS (ESI/Q-TOF) m/z: $[\mathrm{M}+\mathrm{Na}]^{+}$Calcd for $\mathrm{C}_{32} \mathrm{H}_{35} \mathrm{~N}_{5} \mathrm{O}_{6} \mathrm{Na}$ 608.2485; Found 608.2485; Enantiomeric excess were determined by HPLC (Chiralpak IA, Heptane $/ \mathrm{i}-\mathrm{PrOH}=80: 20$, flow rate $=1.0 \mathrm{~mL} \cdot \mathrm{min}^{-1}, 214 \mathrm{~nm}$ ): major diastereoisomer: $\mathrm{t}_{1}=15.4 \mathrm{~min}$ 
(major enantiomer), $\mathrm{t}_{4}=74.5 \mathrm{~min}$ (minor enantiomer); minor diastereoisomer: $\mathrm{t}_{2}=23.9 \min (\operatorname{minor}$ enantiomer), $\mathrm{t}_{3}=26.7 \mathrm{~min}$ (major enantiomer).

Dibenzyl 1-((1R)-2-(((benzyloxy)carbonyl)amino)-1-cyclopropyl-2-(1H-pyrazol-1yl)ethyl)hydrazine-1,2-dicarboxylate (61). Following the general procedure, 61 was obtained from the corresponding (E)-enecarbamate $2 \mathrm{~d}(21.7 \mathrm{mg}, 0.1 \mathrm{mmol})$ and pyrazole $(20 \mathrm{mg}, 0.3 \mathrm{mmol})$. The crude product was purified by column chromatography on silica gel (PE/EtOAc 8:3) to afford 61 as a colorless oil (45 mg, 1:1 dr, 99/95\% ee) in 78\% yield; $d r$ was determined by ${ }^{1} H$ NMR via integration of the singlets at 6.22 and $6.20 \mathrm{ppm} ;[\alpha]_{\mathrm{D}}^{25}-8\left(c 1.0, \mathrm{CH}_{2} \mathrm{Cl}_{2}\right)$; IR (neat) $\vee(\mathrm{cm}-1): 1714,1498,1455$, 1400, 1296, 1260, 1118, 1084, 1051, 1028, 974, 917, 753, 696; ${ }^{1} \mathrm{H}\left\{{ }^{13} \mathrm{C}\right\}$ NMR $\left(500 \mathrm{MHz}, \mathrm{CD}_{3} \mathrm{CN}\right)$ mixture of diastereoisomers and rotamers: $\delta 7.75-6.75(\mathrm{~m}, 19 \mathrm{H}), 6.22(\mathrm{~s}, 1 \mathrm{H}$, diastereoisomer 1), 6.20 (s, 1H, diastereoisomer 2), $5.12-4.75(\mathrm{~m}, 6 \mathrm{H}), 4.00-3.50(\mathrm{~m}, 1 \mathrm{H}), 0.92-0.88(\mathrm{~m}, 1 \mathrm{H}), 0.58-$ $0.08(\mathrm{~m}, 4 \mathrm{H}) ;{ }^{13} \mathrm{C}\left\{{ }^{1} \mathrm{H}\right\} \mathrm{NMR}\left(126 \mathrm{MHz}, \mathrm{CDCl}_{3}\right)$ mixture of diastereoisomers and rotamers: $\delta 156.0$, $155.7,155.5,140.5,140.0,135.9,135.6,130.0,128.6,128.5,128.4,128.3,128.3,127.7,105.5,68.6$ 68.3, 67.9, 67.6, 67.4, 10.6, 5.5, 4.0. HRMS (ESI/Q-TOF) m/z: $[\mathrm{M}+\mathrm{Na}]^{+}$Calcd for $\mathrm{C}_{32} \mathrm{H}_{33} \mathrm{~N}_{5} \mathrm{O}_{6} \mathrm{Na}$ 606.2310; Found 606.2323; enantiomeric excesses were determined by HPLC (Chiralpak IA, Heptane $/ \mathrm{i}-\mathrm{PrOH}=90: 10$, flow rate $=0.9 \mathrm{~mL} / \mathrm{min}, 214 \mathrm{~nm}):$ major diastereoisomer: $\mathrm{t}_{1}=41.5 \mathrm{~min}$ (minor enantiomer), $\mathrm{t}_{2}=56.5 \mathrm{~min}$ (major enantiomer); minor diastereoisomer: $\mathrm{t}_{3}=68.22 \mathrm{~min}$ (major enantiomer), $\mathrm{t}_{4}=99.4 \mathrm{~min}$ (minor enantiomer).

dibenzyl 1-((5S,6S)-5-(1H-indazol-1-yl)-10,10-dimethyl-3-oxo-1,9,9-triphenyl-2,8-dioxa-4-aza-9silaundecan-6-yl)hydrazine-1,2-dicarboxylate (6m). Following the general procedure, 6m was obtained from the corresponding $(E)$-enecarbamate 2 e $(47.3 \mathrm{mg}, 0.1 \mathrm{mmol})$ and indazole $(35.4 \mathrm{mg}$, 
$0.3 \mathrm{mmol}$ ). The crude product was purified by column chromatography on silica gel (PE/EtOAc 8:3) to afford $6 \mathrm{~m}$ as a colorless oil (44 $\mathrm{mg}, 7: 3 d r, 94 / 91 \%$ ee) in $50 \%$ yield; $d r$ was determined by ${ }^{1} H$ $N M R$ via integration of the singlets at 8.02 and $7.98 \mathrm{ppm} ;[\alpha]_{\mathrm{D}}^{25}-20\left(c 1.0, \mathrm{CH}_{2} \mathrm{Cl}_{2}\right) ; \mathrm{IR}(\mathrm{neat}) \mathrm{v}(\mathrm{cm}-$ 1): $1704,1629,1587,1498,1455,1406,1328,1292,1214,1109,1043,1027,976,909,823,736 ;{ }^{1} \mathrm{H}$ $\left\{{ }^{13} \mathrm{C}\right\}$ NMR $\left(500 \mathrm{MHz}, \mathrm{CD}_{3} \mathrm{CN}\right)$ mixture of diastereoisomers and rotamers: $\delta 8.02$ (s, 0.7H, major diastereoisomer), $7.98(\mathrm{~s}, 0.3 \mathrm{H}$, minor diastereoisomer), $7.80-6.73(\mathrm{~m}, 30 \mathrm{H}), 6.66-5.93(\mathrm{~m}, 1 \mathrm{H})$, $5.30-4.56(\mathrm{~m}, 8 \mathrm{H}), 3.90-3.15(\mathrm{~m}, 2 \mathrm{H}), 1.87-1.09(\mathrm{~m}, 4 \mathrm{H}), 1.00(\mathrm{~s}, 9 \mathrm{H}) ; .{ }^{13} \mathrm{C}\left\{{ }^{1} \mathrm{H}\right\} \mathrm{NMR}(126 \mathrm{MHz}$, $\left.\mathrm{CD}_{3} \mathrm{CN}\right)$ mixture of diastereoisomers and rotamers: $\delta 157.4,157.1,156.6,136.4,136.3,135.9,135.3$, $130.7,130.6,129.5,129.4,129.2,129.1,129.0,128.9,128.8,128.7,128.6,128.5,127.9,127.7,127.3$, $127.2,125.3,125.0,122.8,122.7,122.1,122.0,121.8,121.6,121.5,77.9,77.3,68.8,68.2,68.4,68.2$, $67.9,67.7,67.2,67.9,64.8,64.6,64.5,64.3,64.1,63.8,30.1,29.9,27.4,27.3,27.2,27.1,19.8 ;$ HRMS $\left(\right.$ ESI/Q-TOF) m/z: $[\mathrm{M}+\mathrm{Na}]^{+}$Calcd for $\mathrm{C}_{52} \mathrm{H}_{55} \mathrm{~N}_{5} \mathrm{O}_{7} \mathrm{SiNa}$ 912.3746; Found 912.3762; enantiomeric excesses were determined by HPLC (Chiralpak AD-H, Heptane/ $i-\mathrm{PrOH}=90: 10$, flow rate $=1$ $\left.\mathrm{mL} \cdot \mathrm{min}^{-1}, 214 \mathrm{~nm}\right):$ minor diastereoisomer: $\mathrm{t}_{1}=13.85 \mathrm{~min}$ (minor enantiomer), $\mathrm{t}_{4}=48.69$ min (major enantiomer); major diastereoisomer: $\mathrm{t}_{2}=19.99 \min$ (major enantiomer), $\mathrm{t}_{3}=30.28 \min$ (minor enantiomer).

\section{Dibenzyl 1-((5S,6S)-10,10-dimethyl-3-oxo-1,9,9-triphenyl-5-(1H-pyrazol-1-yl)-2,8-dioxa-4-aza-9-} silaundecan-6-yl)hydrazine-1,2-dicarboxylate (6n). Following the general procedure, 6n was obtained from the corresponding $(E)$-enecarbamate $2 \mathrm{e}(47.3 \mathrm{mg}, 0.1 \mathrm{mmol})$ and pyrazole $(20 \mathrm{mg}, 0.3$ mmol). The crude product was purified by column chromatography on silica gel (PE/EtOAc 8:3) to afford 6n as a colorless oil (43 mg, 4:1 dr, 95/91\% ee) in 51\% yield; $d r$ was determined by ${ }^{1} H N M R$ via integration of the multiplets at 5.92 and $5.81 \mathrm{ppm} ;[\alpha]_{\mathrm{D}^{25}}-17\left(\mathrm{c} 1.0, \mathrm{CH}_{2} \mathrm{Cl}_{2}\right) ; \mathrm{IR}$ (neat) v (cm- 
1): $1694,1498,1455,1427,1398,1297,1258,1213,1088,1026,971,911,794 ;{ }^{1} \mathrm{H}\left\{{ }^{13} \mathrm{C}\right\}$ NMR $(500$ $\left.\mathrm{MHz}, \mathrm{CDCl}_{3}\right)$ mixture of diastereoisomers and rotamers: $\delta 7.65-7.61(\mathrm{~m}, 4 \mathrm{H}), 7.41-7.26(\mathrm{~m}, 21 \mathrm{H})$, $7.14(\mathrm{~s}, 2 \mathrm{H}), 6.35-6.06(\mathrm{~m}, 1 \mathrm{H}), 5.95-5.90(\mathrm{~m}, 0.8 \mathrm{H}$, major diastereoisomer), $5.90-5.75(\mathrm{~m}, 0.2 \mathrm{H}$, minor diastereoisomer), $5.39-4.85(\mathrm{~m}, 7.25 \mathrm{H}), 4.60-4.40(\mathrm{~m}, 0.75 \mathrm{H}), 3.89-3.47(\mathrm{~m}, 2 \mathrm{H}), 1.90-$ $1.52(\mathrm{~m}, 4 \mathrm{H}), 1.03(\mathrm{~s}, 9 \mathrm{H}) ;{ }^{13} \mathrm{C}\left\{{ }^{1} \mathrm{H}\right\} \mathrm{NMR}\left(75 \mathrm{MHz}, \mathrm{CDCl}_{3}\right)$ mixture of diastereoisomers and rotamers: $\delta 156.6,156.2,155.5,141.1,140.2,139.8,135.7,134.0,129.7,128.6,128.4,128.3,128.1,127.8$, 127.7, 106.1, 69.3, 68.7, 68.3, 68.0, 67.7, 63.5, 29.2, 28.9, 27.0, 25.0, 19.3; HRMS (ESI/Q-TOF) m/z: $[\mathrm{M}+\mathrm{Na}]^{+}$Calcd for $\mathrm{C}_{48} \mathrm{H}_{53} \mathrm{~N}_{5} \mathrm{O}_{7} \mathrm{SiNa}$ 862.3592; Found 862.3606; enantiomeric excesses were determined by HPLC (Chiralpak OD-H, Heptane $/ i$-PrOH $=95: 5$, flow rate $=0.6 \mathrm{~mL} \cdot \mathrm{min}^{-1}, 214 \mathrm{~nm}$ ): major diastereoisomer: $\mathrm{t}_{1}=22.9 \min$ (minor enantiomer), $\mathrm{t}_{3}=30.0 \mathrm{~min}$ (major enantiomer); minor diastereoisomer: $\mathrm{t}_{2}=26.4 \mathrm{~min}$ (minor enantiomer), $\mathrm{t}_{4}=64.0 \mathrm{~min}$ (major enantiomer).

\section{Dibenzyl 1-((5S,6S)-10,10-dimethyl-5-(4-nitro-1H-pyrazol-1-yl)-3-oxo-1,9,9-triphenyl-2,8-dioxa-4-} aza-9-silaundecan-6-yl)hydrazine-1,2-dicarboxylate (6o). Following the general procedure, 6o was obtained from the corresponding $(E)$-enecarbamate $2 \mathrm{e}(47.3 \mathrm{mg}, 0.1 \mathrm{mmol})$ and 4-nitro-1H-pyrazole (34.0 $\mathrm{mg}, 0.3 \mathrm{mmol}$ ). The crude product was purified by column chromatography on silica gel (PE/EtOAc 8:3) to afford 60 as a colorless oil (46 mg, 4:1 $d r, 99 / 99 \%$ ee) in 52\% yield; $d r$ was determined via integration of the singlets at 8.10 and $7.99 p p m[\alpha]_{\mathrm{D}}{ }^{25}-20\left(c 1.0, \mathrm{CH}_{2} \mathrm{Cl}_{2}\right)$; IR (neat) $v$ (cm-1): 1704, 1515, 1409, 1305, 1257, 1020, 734, 698; ${ }^{1} \mathrm{H}\left\{{ }^{13} \mathrm{C}\right\}$ NMR $\left(500 \mathrm{MHz}, \mathrm{CD}_{3} \mathrm{CN}\right)$ mixture of diastereoisomers and rotamers: $\delta 8.63-8.48(\mathrm{~m}, 1 \mathrm{H}), 8.10(\mathrm{~s}, 0.2 \mathrm{H}$, minor diastereoisomer), $7.99(\mathrm{~s}$, $0.8 \mathrm{H}$, major diastereoisomer), $7.76-7.64(\mathrm{~m}, 4 \mathrm{H}), 7.56-7.21(\mathrm{~m}, 22 \mathrm{H}), 6.08-5.66(\mathrm{~m}, 1 \mathrm{H}), 5.28-$ $4.91(\mathrm{~m}, 6 \mathrm{H}), 4.68-4.50(\mathrm{~m}, 1 \mathrm{H}), 3.70-3.63(\mathrm{~m}, 2 \mathrm{H}), 1.94-1.72(\mathrm{~m}, 2 \mathrm{H}), 1.69-1.51(\mathrm{~m}, 2 \mathrm{H}), 1.02$ $(\mathrm{s}, 9 \mathrm{H}) ;{ }^{13} \mathrm{C}\left\{{ }^{1} \mathrm{H}\right\} \mathrm{NMR}\left(126 \mathrm{MHz}, \mathrm{CDCl}_{3}\right)$ mixture of diastereoisomers and rotamers: $\delta 156.6,155.8$, $155.18,136.68,136.4,136.2,135.9,135.7,135.5,135.5,135.5,135.3,135.0,133.8,133.6,129.8$, 
$129.8,129.7,128.6,128.6,128.5,128.4,128.2,127.8,127.8,127.7,69.1,68.4,68.2,68.0,67.7,67.0$,

63.1, 62.8, 31.5, 29.1, 28.6, 26.9, 26.8, 19.1; HRMS (ESI/Q-TOF) m/z: $[\mathrm{M}+\mathrm{Na}]^{+}$Calcd for $\mathrm{C}_{48} \mathrm{H}_{52} \mathrm{~N}_{6} \mathrm{O} 9 \mathrm{SiNa}$ 907.3416; Found 907.3447; enantiomeric excesses are determined by HPLC (Chiralpak IA, Heptane $/ i-\mathrm{PrOH}=95: 5$, flow rate $=1.0 \mathrm{~mL} \cdot \mathrm{min}^{-1}, 214 \mathrm{~nm}$ ): major diastereoisomer: $\mathrm{t}_{1}=15.6 \mathrm{~min}$ (minor enantiomer), $\mathrm{t}_{3}=48.2 \min$ (major enantiomer); minor diastereoisomer: $\mathrm{t}_{2}=20.7$ min (minor enantiomer), $\mathrm{t}_{4}=60.2 \mathrm{~min}$ (major enantiomer).

\section{Dibenzyl 1-((1S,2R)-1-(((benzyloxy)carbonyl)amino)-1-(7-bromo-1H-indol-3-yl)propan-2-}

yl)hydrazine-1,2-dicarboxylate (6p). Under argon, the (E)-enecarbamate 2a (19.1 mg, $0.1 \mathrm{mmol}, 1.0$ equiv) was dissolved in $\mathrm{CH}_{2} \mathrm{Cl}_{2}(0.4 \mathrm{~mL})$ in a flame-dried flask containing activated powdered $3 \AA$ molecular sieves $(20 \mathrm{mg})$. The solution was stirred at room temperature for $10 \mathrm{~min}$ before being cooled to $-45{ }^{\circ} \mathrm{C}$ and stirred for additional $10 \mathrm{~min}$. Dibenzyl azodicarboxylate $3(44.7 \mathrm{mg}, 0.15$ mmol, 1.5 equiv) dissolved in $\mathrm{CH}_{2} \mathrm{Cl}_{2}(0.3 \mathrm{~mL})$ was added and the reaction mixture was stirred for 10 min. Then EtSH $(7 \mu \mathrm{L}, 0.1 \mathrm{mmol}, 1.0$ equiv) and finally the $(S)$-phosphoric acid 1c $(6 \mathrm{mg}, 0.01$ mmol, 0.1 equiv) as a solution in $\mathrm{CH}_{2} \mathrm{Cl}_{2}(0.3 \mathrm{~mL})$ were added and the reaction mixture was stirred for $16 \mathrm{~h}$ at $-45^{\circ} \mathrm{C}$. Then, 7-bromoindole (58 $\mathrm{mg}, 0.3 \mathrm{mmol}, 3$ equiv) was added and the reaction was stirred at room temperature overnight under $\mathrm{O}_{2}$ atmosphere and blue light irradiation for $16 \mathrm{~h}$. Completion of the reaction was checked by TLC. Solvents were removed in vacuo and the residue was purified by flash column chromatography $\left(\mathrm{CHCl}_{3} / \mathrm{EtOAc}^{95 / 5}\right.$ to $\left.90 / 10\right)$ to give $6 \mathrm{p}$ as a colorless oil (27 mg, > 95:5 $d r, 98 \%$ ee) in 39\% yield.

${ }^{1} \mathrm{H}$ NMR (300 MHz, $\left.\mathrm{CD}_{3} \mathrm{CN}\right) \delta(\mathrm{ppm})$ : (mixture of rotamers) 9.5 (s, rot. 1, 0.1H), $9.13(\mathrm{~s}, 0.9 \mathrm{H}), 7.99$ (brs, 1H), 7.67 (brs, 1H), $7.49-7.23$ (m, 16H), 7.19 (brs, 1H), 6.85 (brs, 1H), 6.09 (s, 1H), $5.35-4.50$ $(\mathrm{m}, 8 \mathrm{H}), 1.27(\mathrm{~d}, J=6.8 \mathrm{~Hz}$, rot. $1,0.3 \mathrm{H}), 1.23(\mathrm{~d}, J=6.5 \mathrm{~Hz}, 3 \mathrm{H})$. According to literature data. ${ }^{18}$ 
Procedure for the large-scale synthesis of dibenzyl 1-((1S,2R)-1-(((benzyloxy)carbonyl)amino)-1(1H-pyrazol-1-yl)propan-2-yl)hydrazine-1,2-dicarboxylate (6a).

Under argon, the benzyl (E)-prop-1-en-1-ylcarbamate 2a (1 mmol, 1.0 equiv, $0.191 \mathrm{~g})$ was dissolved in $\mathrm{CH}_{2} \mathrm{Cl}_{2}(4 \mathrm{~mL})$ in a $25 \mathrm{~mL}$ flame-dried round-bottom flask containing activated powdered $3 \AA$ molecular sieves $(0.2 \mathrm{~g})$. The solution was stirred at room temperature for $10 \mathrm{~min}$ before being cooled to $-45^{\circ} \mathrm{C}$ and stirred for additional $10 \mathrm{~min}$. Dibenzyl azodicarboxylate ( $1.5 \mathrm{mmol}, 1.5$ equiv, $0.45 \mathrm{~g})$ dissolved in $\mathrm{CH}_{2} \mathrm{Cl}_{2}(3 \mathrm{~mL})$ was added and the reaction mixture was stirred for $10 \mathrm{~min}$. Then EtSH $(1 \mathrm{mmol}, 1$ equiv, $77 \mu \mathrm{L})$ and finally the chiral photocatalyst $1 \mathrm{c}(0.1 \mathrm{mmol}, 0.1$ equiv, $60 \mathrm{mg})$ were added as a solution in $\mathrm{CH}_{2} \mathrm{Cl}_{2}(3 \mathrm{~mL})$ and the reaction mixture was stirred for $16 \mathrm{~h}$ at $-45^{\circ} \mathrm{C}$. Then, the pyrazole was added ( 3 mmol, 3 equiv, $0.204 \mathrm{~g}$ ) and the reaction was stirred at room temperature under an $\mathrm{O}_{2}$ atmosphere in PhotoRedOx Box (Evoluchem) with a 30 W blue LED (EvoluChem, model: HCK101201-008, wavelength $405 \mathrm{~nm}$, LED: CREE XPE) for $24 \mathrm{~h}$. Completion of the reaction was checked by TLC. The solvent was evaporated under reduced pressure and the residue was purified by flash column chromatography (PE/EtOAc 8:3) to afford 6a as a colourless oil $(0.49 \mathrm{~g}, 7: 3 d r, 98 / 97 \% e e)$ in $88 \%$ yield.

\section{ASSOCIATED CONTENT}

\section{Supporting Information}

The Supporting Information is available free of charge at https://pubs.acs.org/ and contains electrochemical data, UV-vis data, and ${ }^{1} \mathrm{H},{ }^{13} \mathrm{C}$ NMR and HPLC spectra for all new compounds.

\section{AUTHOR INFORMATION}




\section{Corresponding Author}

Géraldine Masson, Institut de Chimie des Substances Naturelles, CNRS UPR 2301, Université ParisSaclay, 91198 Gif-sur-Yvette Cedex, France. https://orcid.org/0000-0003-2333-7047, E-mail: geraldine.masson@cnrs.fr

\section{Other Authors}

Jiyuan Lyu, Institut de Chimie des Substances Naturelles, CNRS UPR 2301, Université Paris-Saclay, 91198 Gif-sur-Yvette Cedex, France.

Aurélie Claraz, Institut de Chimie des Substances Naturelles, CNRS UPR 2301, Université ParisSaclay, 91198 Gif-sur-Yvette Cedex, France.

Maxime Vitale, Laboratoire des biomolécules, LBM, Département de chimie, École normale supérieure, PSL University, Sorbonne Université, CNRS, 75005 Paris, France. https://orcid.org/00000002-6740-2472

Clémence Allain, PPSM, ENS Paris-Saclay, CNRS UMR 8531, 61, Avenue du Président Wilson, 94235 Cachan cedex, France. https://orcid.org/0000-0002-2908-264X.

\section{NOTE}

The authors declare no competing financial interest.

\section{AUTHOR CONTRIBUTIONS}

All authors have given approval to the final version of the manuscript.

\section{ACKNOWLEDGMENT}

We thank ICSN and CNRS for financial support. JL thanks china scholarship council for the doctoral fellowship.

\section{REFERENCES}

(1) For recent reviews on chiral phosphoric acid catalysis: (a) Akiyama, T. Stronger Brønsted Acids. Chem. Rev. 2007, 107, 5744. (b) Terada, M. Chiral phosphoric acids as versatile catalysts for 
enantioselective transformations. Synthesis 2010, 1929. (c) Rueping, M.; Kuenkel, A.; Atodiresei, I. Chiral Brønsted acids in enantioselective carbonyl activations - activation modes and applications. Chem. Soc. Rev. 2011, 40, 4539. (d) Schenker, S.; Zamfir, A.; Freund, M.; Tsogoeva, S. B. Developments in chiral binaphthyl-derived Brønsted/Lewis acids and hydrogen-bond-donor organocatalysis. Eur. J. Org. Chem. 2011, 2209. (e) Parmar, D.; Sugiono, E.; Raja, S.; Rueping, M. Complete field guide to asymmetric BINOL-phosphate derived Brønsted acid and metal catalysis: history and classification by mode of activation; Brønsted acidity, hydrogen bonding, ion pairing and metal phosphates. Chem. Rev. 2017, 117, 10608; Chem. Rev. 2014, 114, 9047. (f) Merad, J.; Lalli, C.; Bernadat, G.; Maury, J.; Masson, G. Enantioselective Brønsted acid catalysis as a tool for the synthesis of natural products and pharmaceuticals. Chem. Eur. J. 2018, 24, 3925. (g) Gualandi, A.; Rodeghiero, G.; Cozzi, P. G. Catalytic stereoselective SN 1-Type reactions promoted by chiral phosphoric acids as Brønsted acid catalysts. Asian J. Org. Chem. 2018, 7, 1957. (h) Maji, R.; Mallojjala, S. C.; Wheeler, S. E. Chiral phosphoric acid catalysis: from numbers to insights. Chem. Soc. Rev. 2018, 47, 1142.

(2) For selected recent reviews on photoredox catalysis, see: (a) Prier, C. K.; Rankic, D. A.; MacMillan, D. W. Visible Light Photoredox Catalysis with transition metal complexes: applications in organic synthesis. Chem. Rev. 2013, 113, 5322. (b) Ravelli, D.; Fagnoni, M.; Albini, A. Photoorganocatalysis. What For? Chem. Soc. Rev. 2013, 42, 97. (c) Hopkinson, M. N.; Sahoo, B.; Li, J.-L.; Glorius, F. Dual Catalysis sees the light: combining photoredox with organo-, acid, and transition-metal catalysis. Chem. Eur. J. 2014, 20, 3874. (d) Romero, N. A.; Nicewicz, D. A. Organic photoredox catalysis Chem. Rev. 2016, 116, 10075. (e) Shaw, M. H.; Twilton, J.; MacMillan, D. W. C. Photoredox catalysis in organic chemistry. J. Org. Chem. 2016, 81, 6898. (f) Skubi, K. L.; Blum, 
T. R.; Yoon, T. P. Dual catalysis strategies in photochemical synthesis Chem. Rev. 2016, 116, 10035.

(g) Marzo, L.; Pagire, S.K.; Reiser, O.; König, B.; Visible-light photocatalysis: does it make a difference in organic synthesis Angew. Chem., Int. Ed. 2018, 57, 10034. (h) Sideri, I. K.; Voutyritsa, E.; Kokotos, C. G. Photoorganocatalysis, small organic molecules and light in the service of organic synthesis: the awakening of a sleeping giant. Org. Biomol. Chem. 2018, 16, 4596.

(3) Selected recent reviews (a) Brimioulle, R.; Lenhart, D.; Maturi, M. M.; Bach, T. Enantioselective Catalysis of Photochemical Reactions. Angew. Chem., Int. Ed. 2015, 54, 3872. (b) Meggers, E. Asymmetric catalysis activated by visible light. Chem. Commun. 2015, 51, 3290. (c) Wang, C.; Lu, Z. Catalytic enantioselective organic transformations via visible light photocatalysis. Org. Chem. Front. 2015, 2, 179. (d) SOMO and photoredox asymmetric organocatalysis. Vitale, M.R. in radical and ion-pairing strategies in asymmetric organocatalysis, Masson, G., Malacria, M. Eds.; ISTE Press: London 2016, p 1. (e) Jiang, C.; Chen, W.; Zheng, W.-H.; Lu, H. Advances in asymmetric visible-light photocatalysis, 2015-2019. Org. Biomol. Chem. 2019, 17, 8673. (f) Silvi, M.; Melchiorre, P. Enhancing the potential of enantioselective organocatalysis with light. Nature, 2018, 554, 41. (g) Hong, B.-C. Enantioselective synthesis enabled by visible light photocatalysis. Org. Biomol. Chem. 2020, 18, 4298.

(4) Nicewicz, D. A.; MacMillan, D. W. C. Merging photoredox catalysis with organocatalysis: the direct asymmetric alkylation of aldehydes Science 2008, 322, 77.

(5) First example of catalytic enantioselective chemistry merging hydrogen bonding organocatalysis with UV-light photocatalysis, see: A. Bauer, A.; Westkämper, F.; Grimme, S.; Bach, T. Catalytic enantioselective reactions driven by photoinduced electron transfer. Nature 2005, 436, 
(6) (a) Alonso, R.; Bach, T. A chiral thioxanthone as an organocatalyst for enantioselective [2 + 2] photocycloaddition reactions induced by visible light. Angew. Chem., Int. Ed. 2014, 53, 4368. (b) Tröster, A.; Alonso, R.; Bauer, A.; Bach, T. Enantioselective intermolecular $[2+2]$ photocycloaddition reactions of 2(1H)-quinolones induced by visible light irradiation. J. Am. Chem. Soc. 2016, 138, 7808. (c) Hölzl-Hobmeier, A.; Bauer, A.; Silva, A. V.; Huber, S. M.; Bannwarth, C.; Bach, T. Catalytic deracemization of chiral allenes by sensitized excitation with visible light. Nature 2018, 564, 240. (d) Mayr, F.; Mohr, L.-M.; Rodriguez, E.; Bach, T. Synthesis of chiral thioureathioxanthone hybrids. Synthesis 2017, 49, 5238. (e) Rigotti, T.; Casado-Sánchez, A.; Cabrera, S.; Pérez-Ruiz, R.; Liras, M.; de la Peña O’Shea, V. A.; Alemán, J. A bifunctional photoaminocatalyst for the alkylation of aldehydes: design, analysis, and mechanistic studies. ACS Catal. 2018, 8, 5928.

(7) See also selected examples of chiral metal photocatalysts: (a) Huo, H.; Shen, X.; Wang, C.; Zhang, L.; Röse, P.; Chen, L.- A.; Harms, K.; Marsch, M.; Hilt, G.; Meggers, E. Asymmetric photoredox transition-metal catalysis activated by visible light. Nature 2014, 515, 100. (b) Skubi, K. L.; Kidd, J. B.; Jung, H.; Guzei, I. A.; Baik, M.-H.; Yoon, T. P. Enantioselective excited-state photoreactions controlled by a chiral hydrogen-bonding Iridium sensitizer. J. Am. Chem. Soc. 2017, 139, 17186. (c) Ding, W.; Lu, L.-Q.; Zhou, Q.-Q.; Wei, Y.; Chen, J.-R.; Xiao, W.-J. Bifunctional photocatalysts for enantioselective aerobic oxidation of $\beta$-Ketoesters. J. Am. Chem. Soc. 2017, 139, 63. (d) Huang, X.; Lin, J.; Shen, T.; Harms, K.; Marchini, M.; Ceroni, P.; Meggers, E. Asymmetric [3 +2] photocycloadditions of cyclopropanes with alkenes or alkynes through visible-light excitation of catalyst-bound substrates. Angew. Chem., Int. Ed. 2018, 57, 5454. (e) Schweitzer-Chaput, B.; 
Horwitz, M A.; de Pedro Beato, E.; Melchiorre, P. Photochemical generation of radicals from alkyl electrophiles using a nucleophilic organic catalyst Nat. Chem. 2019, 11, 129. (f) Zheng, J.; Swords, W. B.; Jung, H.; Skubi, K. L; Kidd, J. B; Meyer, G. J.; Baik, M.-H.; Yoon, T. P. Enantioselective intermolecular excited-state photoreactions using a chiral Ir triplet sensitizer: separating association from energy transfer in asymmetric photocatalysis. J. Am. Chem. Soc. 2019, 141, 13625.

(8) (a) He, L.; Laurent, G.; Retailleau, P.; Folleas, B.; Brayer, J.-L.; Masson, G. Highly enantioselective aza-Diels-Alder reaction of 1-azadienes with enecarbamates catalyzed by chiral phosphoric acids. Angew. Chem., Int. Ed. 2013, 52, 11088. (b) J. Pous, T. Courant, G. Bernadat, B. I. Ioga, F. Blanchard, G. Masson, Regio-, diastereo-, and enantioselective nitroso-Diels-Alder reaction of 1,3-diene-1-carbamates catalyzed by chiral phosphoric acids J. Am. Chem. Soc. 2015, 137, 11950. (c) Dumoulin, A.; Lalli, C.; Retailleau, P.; Masson, G. Catalytic, highly enantioselective, direct amination of enecarbamates. Chem. Commun. 2015, 51, 5383. (d) Dumoulin, A.; Bernadat, G.; Masson, G. Enantioselective Three-Component Amination of Enecarbamates Enables the Synthesis of Structurally Complex Small Molecules. J. Org. Chem. 2017, 82, 1775. (e) Gelis, C.; Levitre, G.; Merad, J.; Retailleau, P.; Neuville, L.; Masson, G. Highly Diastereo- and enantioselective synthesis of cyclohepta[b]indoles by chiral-phosphoric-acid-catalyzed (4+3) cycloaddition. Angew. Chem., Int. Ed. 2018, 57, 12121. (f) Jarrige, L.; Glavač, D.; Levitre, G.; Retailleau, P.; Bernadat, G.; Neuville, L.; Masson, G. Chiral phosphoric acid-catalyzed enantioselective construction of structurally diverse benzothiazolopyrimidines. Chem. Sci. 2019, 10, 3765. (g) Varlet, T.; Gelis, C.; Retailleau, P; Bernadat, G.; Neuville, L; Masson, G. Enantioselective redox-divergent chiral phosphoric acid catalyzed quinone Diels-Alder reactions. Angew. Chem., Int. Ed. 2020, 59, 8491. 
(9) (a) Courant, T.; Masson, G. Photoredox initiated $\alpha$-alkylation of imines via a three-component radical/cationic reaction. Chem. Eur. J. 2012, 18, 423. (b) Lebée, C.; Languet, M.; Allain, C.; Masson, G. Photoredox-catalyzed three-component tandem process: an assembly of complex trifluoromethylated phthalans and isoindolines. Org. Lett. 2016, 18, 1478. (c) Jarrige, L.; Levitre, G.; Masson, G. Visible-light photoredox-catalyzed coupling reaction of azoles with $\alpha$-carbamoyl sulfides. J. Org. Chem. 2016, 81, 7230. (d) Lanzi, M.; Merad, J.; Boyarskaya, D.; Maestri, G.; Allain, C.; Masson, G. Visible light-triggered C-C and C-N bonds formation by C-S bonds cleavage of benzylic thioethers. Org. Lett. 2018, 20, 5247. (e) Le, T.; Courant, T.; Merad, J.; Allain, C.; Audebert, P.; Masson, G. s-Tetrazine dyes: a facile generation of photoredox organocatalysts for routine oxidations. J. Org. Chem. 2019, 84, 16139. (f) Levitre, G.; Dagousset, G.; Anselmi, E.; Tuccio, B.; Magnier, E.; Masson, G. Four-component photoredox-mediated azidoalkoxy-trifluoromethylation of alkenes. Org. Lett. 2019, 21, 6005.

(10) (a) Fogg, D. E.; Santos, E. N. Tandem catalysis: a taxonomy and illustrative review. Coord. Chem. Rev. 2004, 248, 2365. (b) Ajamain, A.; Gleason, J. Two birds with one metallic stone: single-pot catalysis of fundamentally different transformations. Angew. Chem., Int. Ed. 2004, 43, 3754. (c) Wasilke, J.-C.; Obrey, S. J.; Baker, R. T.; Bazan, G. C. Concurrent tandem catalysis. Chem. Rev. 2005, 105, 1001. (d) Lee, J. M.; Na, J.; Han, H.; Chang, S. Cooperative multi-catalyst systems for one-pot organic transformations. Chem. Soc. Rev. 2004, 33, 302.

(11) (a) Mayr, F.; Brimioulle, R.; Bach, T. A Chiral Thiourea as a Template for Enantioselective Intramolecular [2 + 2] Photocycloaddition Reactions. J. Org. Chem. 2016, 81, 6965. (b) Tripathi, C. B.; Ohtani, T.; Corbett, M. T.; Ooi, T. Photoredox ketone catalysis for the direct C-H imidation and 
acyloxylation of arenes. Chem. Sci. 2017, 8, 5622.

(12) Pecho, F.; Zou, Y.-Q.; Gramüller, J.; Mori, T.; Huber, S. M.; Bauer, A.; Gschwind, R. M.; Bach, T. A thioxanthone sensitizer with a chiral phosphoric acid binding site: properties and applications in visible light-mediated cycloadditions. Chem. Eur. J. 2020, 26, 5190.

(13) Hatano, M.; Horibe, T.; Ishihara, K. Chiral lithium(I) binaphtholate Salts for the enantioselective direct Mannich-type reaction with a change of Syn/Anti and absolute stereochemistry. J. Am. Chem. Soc. 2010, 132, 56.

(14) Gtz, C.; Hovorka, R.; Schnakenburg, G.; Lützen, A. Homochiral supramolecular $\mathrm{M}_{2} \mathrm{~L}_{4}$ cages by high-fidelity self-sorting of chiral ligands. Chem. Eur. J. 2013, 19, 10890.

(15) (a) Yonesaki, R.; Kondo, Y.; Akkad, W.; Sawa, M.; Morisaki, K.; Morimoto, H.; Ohshima, T. 3-Mono-substituted BINOL phosphoric acids as effective organocatalysts in direct enantioselective Friedel-Crafts-type alkylation of N-unprotected $\alpha$-ketiminoester. Chem. Eur. J. 2018, 24, 15211. (b) Hashimoto, T.; Galvez, A. O.; Maruoka, K. Boronic acid-catalyzed, highly enantioselective azaMichael additions of hydroxamic acid to quinone imine ketals. J. Am. Chem. Soc. 2015, 137, 16016.

(16) 1,2-dichloroethane (DCE) was used in lieu of dichloromethane in order to prevent variation of concentration over time.

(17) Calculated using the Rehm-Weller equation: Rehm, D., Weller, A. Kinetics of fluorescence quenching by electron and $\mathrm{H}$-atom transfer. Isr. J. Chem., 1970, 8, 259.

(18) Levitre, G.; Audubert, C.; Dumoulin, A.; Goual, N.; Retailleau, P.; Moreau, X.; Masson, G. Combining Organocatalysis and Photoredox Catalysis: An Asymmetric Synthesis of chiral $\beta$-amino 


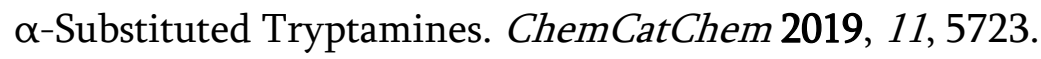

(19) Schiza, A.; Spiliopoulou, N.; Shahua, A.; Kokotos, C. G. Combining organocatalysis with photoorganocatalysis: photocatalytic hydroacylation of asymmetric organocatalytic Michael addition products. New J. Chem. 2018, 42, 18844.

(20) Selected examples, see: (a) Yu, J.-S.; Wua Y.B.; Zhou, F. The first catalytic asymmetric thioacetalization by chiral phosphoric acid catalysis. Org. Biomol. Chem. 2016, 14, 2205. (b) Zhang, L.; Xiang, S.; Wang, J.; Wang, J.; Xiao, J.; Wang, J-Q. Phosphoric acid-catalyzed atroposelective construction of axially chiral arylpyrroles. Nat Commun 2019, 10, 566.

(21) The direct three-component amination of $2 \mathbf{a}$ with azodicarboxylate $3 \mathbf{a}$ and pyrazole $5 \mathbf{a}$ in the presence of 14 , leads to nearly racemic product $6 \mathrm{a}(18 \%$ ee).

(22) (a) Masuda, Y.; Ishida, N.; Murakami, M. Aryl ketones as single-electron-transfer photoredox catalysts in the nickel-catalyzed homocoupling of aryl halides. Eur. J. Org. Chem. 2016, 5822. (b) Ye, C., Zhang, Y., Ding, A. et al. Visible light sensitizer-catalyzed highly selective photo oxidation from thioethers into sulfoxides under aerobic condition. Sci. Rep. 2018, 8, 2205.

(23) Abdullah, K. A.; Kemp, T. J. Quenching of excited-state xanthone and thioxanthone by inorganic anions. J. Chem. Soc., Perkin Trans. 2, 1985, 1279.

(24) The formation of $\mathrm{H}_{2} \mathrm{O}_{2}$ generated by disproportionation of hydroperoxyl radical $\left(\mathrm{HO}_{2} \bullet\right)$ was previously demonstrated by a potassium iodide—starch indicator test, see ref 9e.

(25) The radical chain propagation initiated by thiyl radical 20 or hydroperoxy radical HOO $\bullet$ 
not the predominant mechanistic pathway.

(26) Michelin, C.; Hoffmann, N. Photosensitization and photocatalysis-perspectives in organic synthesis. ACS Catal. 2018, 8, 12046.

(27) 6a was obtained in a comparable yield (75\%) and selectivity (7:3 dr, 98/98 ee) when the reaction was performed in DCE instead of $\mathrm{CH}_{2} \mathrm{Cl}_{2}$.

(28) Phosphoric acid itself can slowly activate 6a (entry 7, Table 2) and this may account for the small amounts of product $6 \mathrm{a}$ formed when the reaction is conducted in the absence of $\mathrm{O}_{2}$

(29) Carboni, A.; Dagousset, G.; Magnier, E.; Masson, G. Photoredox-induced three-component oxy-, amino-, and carbotrifluoromethylation of enecarbamates. Org. Lett. 2014, 16, 1240. 